\title{
Gráficas de Superficies Cuádricas y trazas empleando GeoGebra
}

\author{
Angie Solís Palma \\ ansolis@itcr.ac.cr \\ Escuela de Matemática \\ Instituto Tecnológico de Costa Rica
}

\begin{abstract}
Resumen. En este artículo se pretende mostrar de una manera gráfica y dinámica, las diferentes trazas que se pueden realizar sobre una superficie cuádrica, cuando ésta es intersecada por un plano paralelo a alguno de los planos coordenados y su implementación en el programa GeoGebra.

Palabras clave: Traza, superficie, cuádrica, parametrización, GeoGebra

Abstract. This article is intended to show in a graphic and dynamic way, the different traces that can be performed on a quadric surface, where it is intersected by a plane parallel to one of the coordinate planes and their implementation in the program GeoGebra.
\end{abstract}

KeyWords: Trace, surface, cuadric, parametrization, Geogebra

\subsection{Introducción}

El objetivo de este documento es aportar a los estudiantes del campo de la ingeniería unos ejemplos básicos, de cómo sacar el mayor provecho a Geogebra en la graficación de trazas y superficies, utilizando diferentes tipos de parametrizaciones, así como, mostrar mediante un applet la visualización de las trazas de una superficie cuádrica desde diferentes ángulos. 


\subsection{Cuádricas}

Nos interesan las superficies de ecuación $z=f(x, y)$ o $F(x, y, z)=0$, es decir, las superficies formadas por los puntos $(x, y, z)$ que satisfacen la ecuación $z=f(x, y)$ o $F(x, y, z)=0$.

En particular trabajaremos únicamente con superficies cuádricas cuyas ecuaciones están dadas en forma canónica.

Existen seis tipos básicos de superficies cuádricas: Elipsoide, Hiperboloide de una hoja, Hiperboloide de dos hojas, Cono Elíptico, Paraboloide Elíptico y Paraboloide Hiperbólico.

\section{Elipsoide}

\section{Definición 1.1}

La ecuación canónica del elipsoide centrado en $C$ es:

$$
\frac{(x-i)^{2}}{a^{2}}+\frac{(y-j)^{2}}{b^{2}}+\frac{(z-k)^{2}}{c^{2}}=1
$$

donde $C(i, j, k)$ es un punto en el espacio y $a, b, c$ son constantes reales positivas.

\section{Ejemplo 1.1}

Considere el elipsoide de ecuación:

$$
\frac{(x-1)^{2}}{4}+\frac{(y-3)^{2}}{1}+\frac{(z-1)^{2}}{9}=1
$$

A continuación se puede encontrar su representación gráfica:

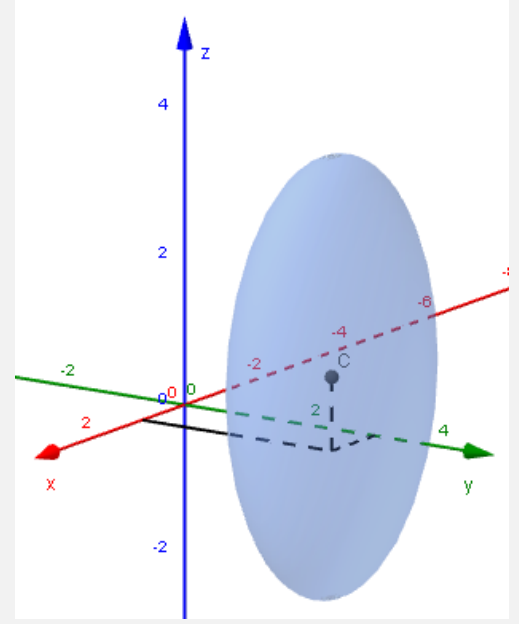

Gráficas de Superficies Cuádricas y trazas empleando GeoGebra . Angie Solís Palma

Derechos Reservados (C) 2015 Revista digital Matemática, Educación e Internet (http://tecdigital.tec.ac.cr/revistamatematica/) 


\section{Definición 1.2}

Si $S$ es una superficie en el espacio de ecuación $F(x, y, z)=0$, se llaman trazas de las superfice a las curvas:
a.) $F(x, y, c)=0, z=c$
b.) $F(x, c, z)=0, y=c$
c.) $F(c, y, z)=0, x=c$

\section{Ejemplo 1.2}

Considere el elipsoide de ecuación $\frac{(x-1)^{2}}{4}+\frac{(y-3)^{2}}{1}+\frac{(z-1)^{2}}{9}=1$. Calcule y dibuje las trazas que se obtienen con los planos $x=1, y=3$ y $z=1$.

\section{Solución:}

$$
\text { Traza } x=1
$$

$$
\text { Elipse: } \frac{(y-3)^{2}}{1}+\frac{(z-1)^{2}}{9}=1
$$

Elipse: $\frac{(x-1)^{2}}{4}+\frac{(z-1)^{2}}{9}=1$

Elipse: $\frac{(x-1)^{2}}{4}+\frac{(y-3)^{2}}{1}=1$
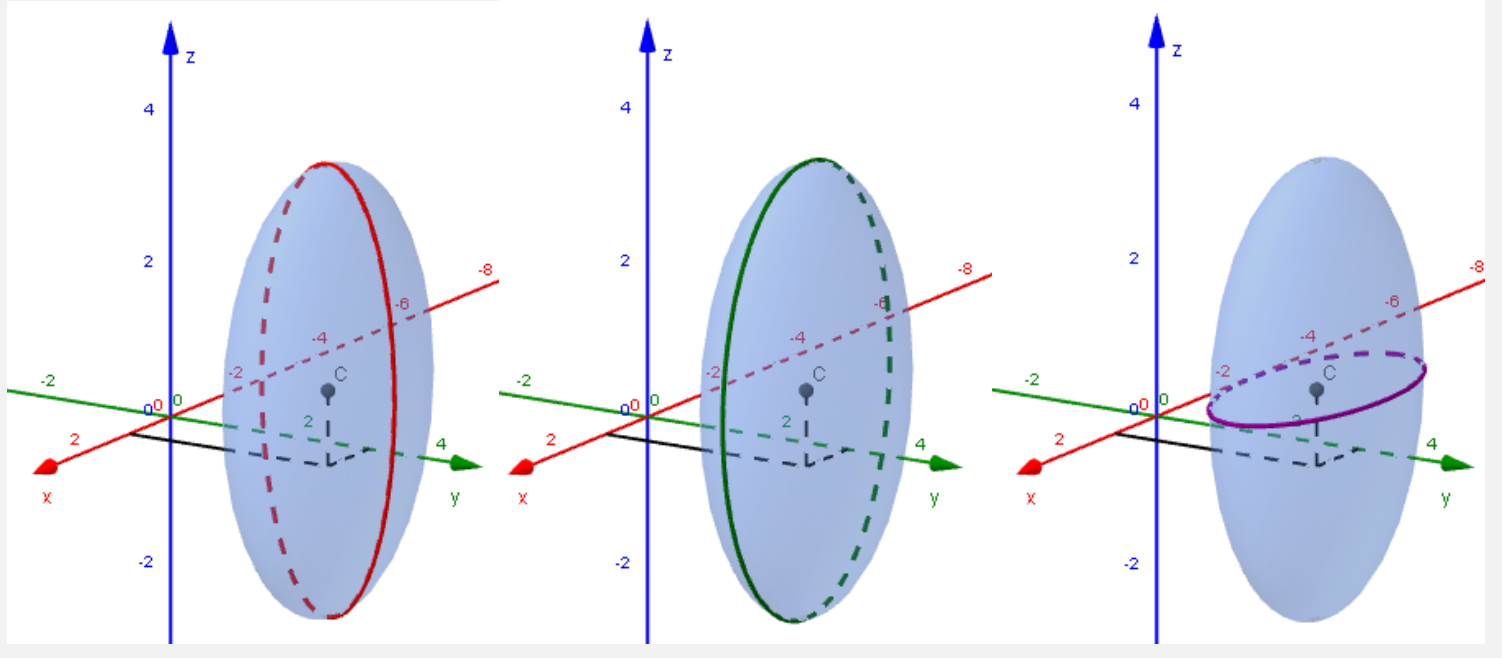

Nota: Recuerde que las trazas son solo las curvas representadas en rojo, verde y morado, en las gráficas anteriores se presenta también la superficie para comprender mejor la traza.

\section{Applet para las trazas de un elipsoide}

En el siguiente applet, se pueden manipular los valores de los parámetros, para las constantes $a, b$ y $c$ de los denominadores, para esto deslice los puntos a la derecha de cada parámetro sobre las líneas. También deslice los puntos sobre las líneas a la derecha de $i, j$ y $k$ para cambiar el centro del elipsoide. 
Para poder observar el plano y las trazas sobre el elipsoide, debe seleccionar, dando click, en la traza que desea y manipular los valores de las trazas deslizando los puntos $x, y$ o $z$, según la traza seleccionada.

Por otra parte, si desea tener otra vista del elipsoide, de click sobre la gráfica y manteniendo presionado el ratón, deslice hacia arriba, abajo, derecha o izquierda el sistema de coordenadas para girarlo.

Para ingresar al applet, debe dar click sobre la siguiente imagen o ingresar en el link: Applet Elipsoide.
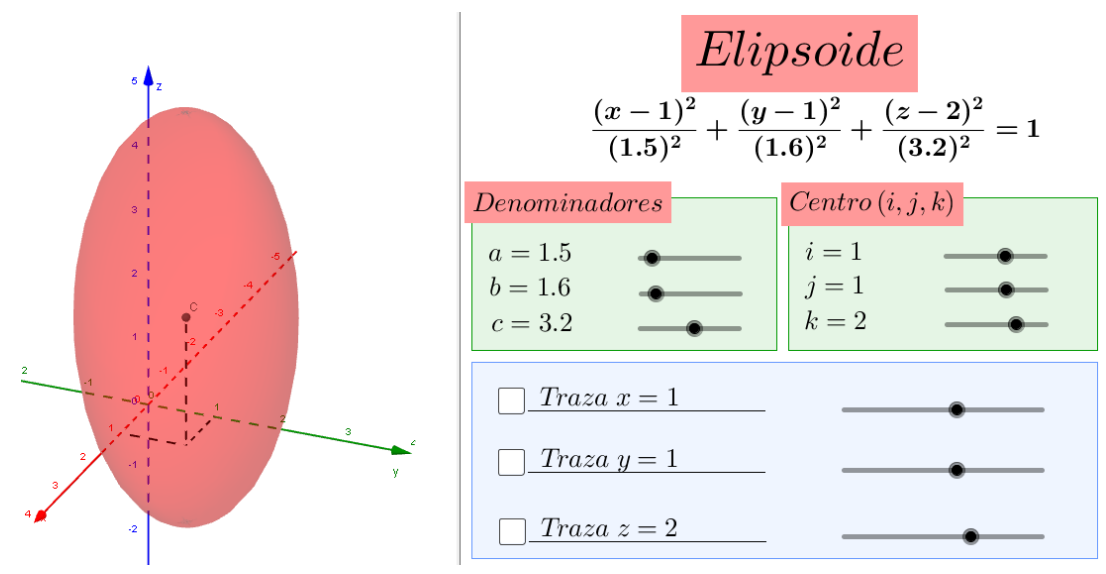

\section{Actividad}

Considere la superficie cuádrica $S$ de ecuación:

$$
\frac{(x-1)^{2}}{4}+\frac{(y-1)^{2}}{4}+z^{2}=1
$$

Calcule y dibuje las trazas que se obtienen con los planos $x=1, y=1$ y $z=0$.

\section{Actividad}

Considere la superficie cuádrica $F$ de ecuación:

$$
\frac{(x+2)^{2}}{9}+\frac{y^{2}}{3}+(z+1)^{2}=1
$$

Calcule y dibuje las trazas que se obtienen con los planos $x=-2, y=0$ y $z=-1$. 


\section{Hiperboloide de una hoja}

\section{Definición 1.3}

La ecuación canónica del hiperboloide de una hoja centrado en $C$, con eje en z es:

$$
\frac{(x-i)^{2}}{a^{2}}+\frac{(y-j)^{2}}{b^{2}}-\frac{(z-k)^{2}}{c^{2}}=1
$$

donde $C(i, j, k)$ es un punto en el espacio y $a, b, c$ son constantes reales positivas.

El eje del hiperboloide de una hoja corresponde a la variable cuyo coeficiente es negativo.

\section{Ejemplo 1.3}

Considere el hiperboloide de una hoja de ecuación:

$$
\frac{(x+1)^{2}}{1}+\frac{y^{2}}{4}-\frac{(z-1)^{2}}{9}=1
$$

A continuación se puede encontrar su representación gráfica:

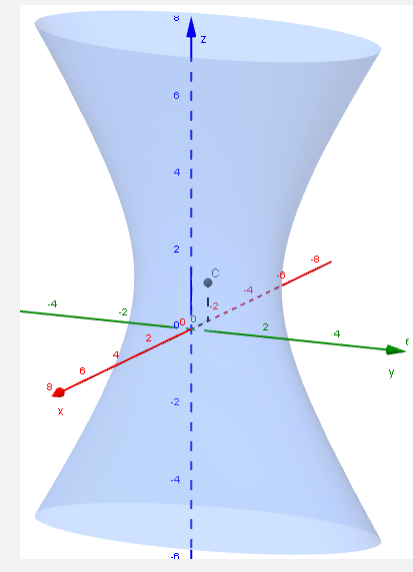

\section{Ejemplo 1.4}

Considere el hiperboloide de una hoja de ecuación:

$$
\frac{(x+1)^{2}}{1}+\frac{y^{2}}{4}-\frac{(z-1)^{2}}{9}=1
$$

Calcule y dibuje las trazas que se obtienen con los planos $x=-1, y=0, z=1, z=4$ y $z=-2$.

\section{Solución:}




$$
\text { Traza } x=-1
$$

Hipérbola: $\frac{y^{2}}{4}-\frac{(z-1)^{2}}{9}=1$

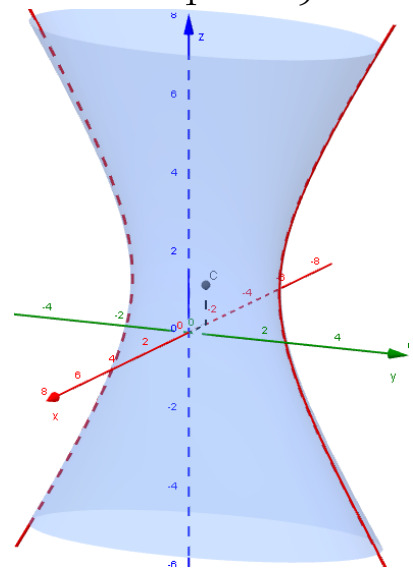

Traza $y=0$

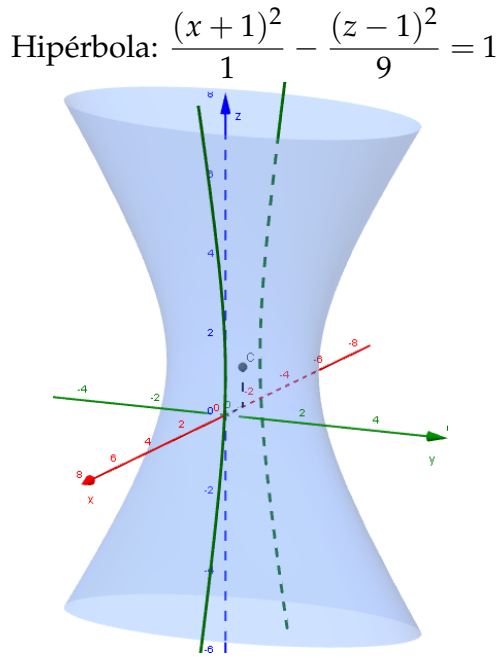

Traza $z=1$

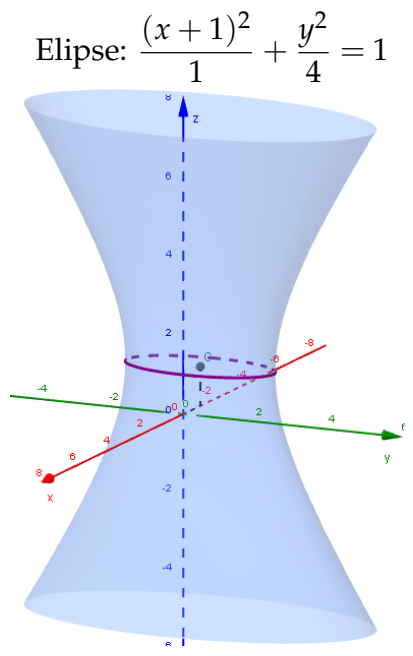

Traza $z=-2$
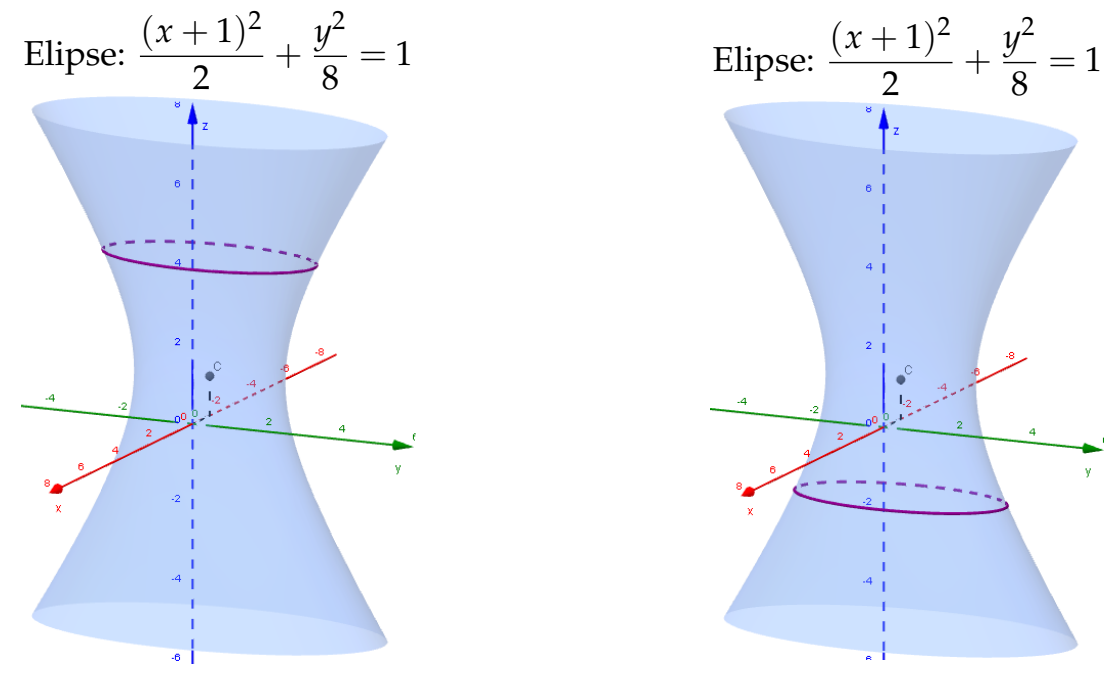

Nota: Recuerde que las trazas son solo las curvas representadas en rojo, verde y morado.

\section{Applet para las trazas de un hiperboloide de una hoja}

El siguiente applet, se pueden manipular de igual manera que el anterior.

Para ingresar al applet, debe dar click sobre la siguiente imagen o ingresar en el link: Applet Hiperboloide de una hoja. 


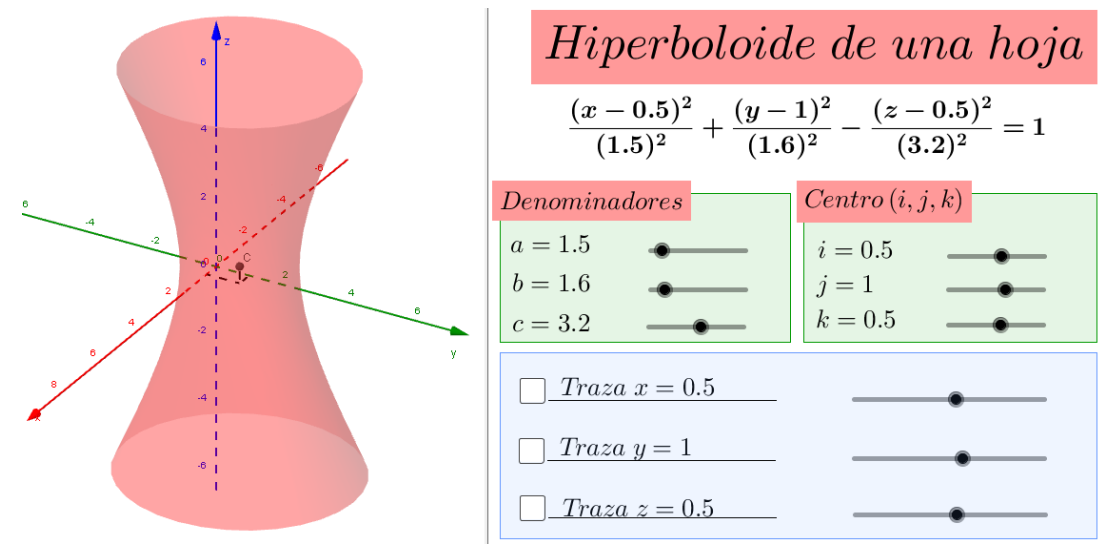

\section{Actividad}

Considere la superficie cuádrica $S$ de ecuación:

$$
\frac{(x-2)^{2}}{9}+\frac{(y-1)^{2}}{2}-(z+2)^{2}=1
$$

Calcule y dibuje las trazas que se obtienen con los planos $x=2, y=1, z=-1$ y $z=-3$.

\section{Actividad}

Considere la superficie cuádrica $H$ de ecuación:

$$
(x+5)^{2}-\frac{y^{2}}{4}+\frac{(z+2)^{2}}{4}=1
$$

Calcule y dibuje las trazas que se obtienen con los planos $x=-5, y=2, y=-2$ y $z=-2$.

\section{Hiperboloide de dos hojas}

\section{Definición 1.4}

La ecuación canónica del hiperboloide de dos hojas centrado en $C$, con eje en z es:

$$
-\frac{(x-i)^{2}}{a^{2}}-\frac{(y-j)^{2}}{b^{2}}+\frac{(z-k)^{2}}{c^{2}}=1
$$

donde $C(i, j, k)$ es un punto en el espacio y $a, b, c$ son constantes reales positivas.

El eje del hiperboloide de dos hojas corresponde a la variable cuyo coeficiente es positivo. 


\section{Ejemplo 1.5}

Considere el hiperboloide de dos hojas de ecuación:

$$
-\frac{(x-3)^{2}}{4}-\frac{(y-1)^{2}}{4}+\frac{(z-1)^{2}}{9}=1
$$

A continuación se puede encontrar su representación gráfica:

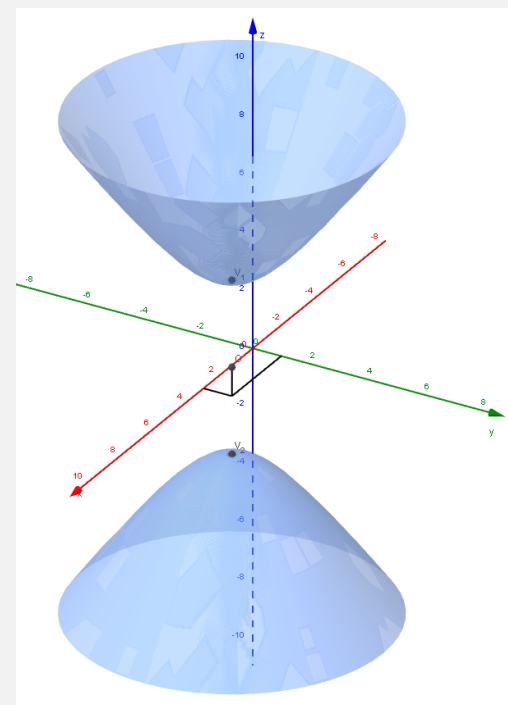

\section{Ejemplo 1.6}

Considere el hiperboloide de dos hojas de ecuación:

$$
-\frac{(x-3)^{2}}{4}-\frac{(y-1)^{2}}{4}+\frac{(z-1)^{2}}{9}=1
$$

Calcule y dibuje las trazas que se obtienen con los planos $x=3, y=1, z=7$ y $z=-5$.

\section{Solución:}




$$
\text { Traza } x=3
$$

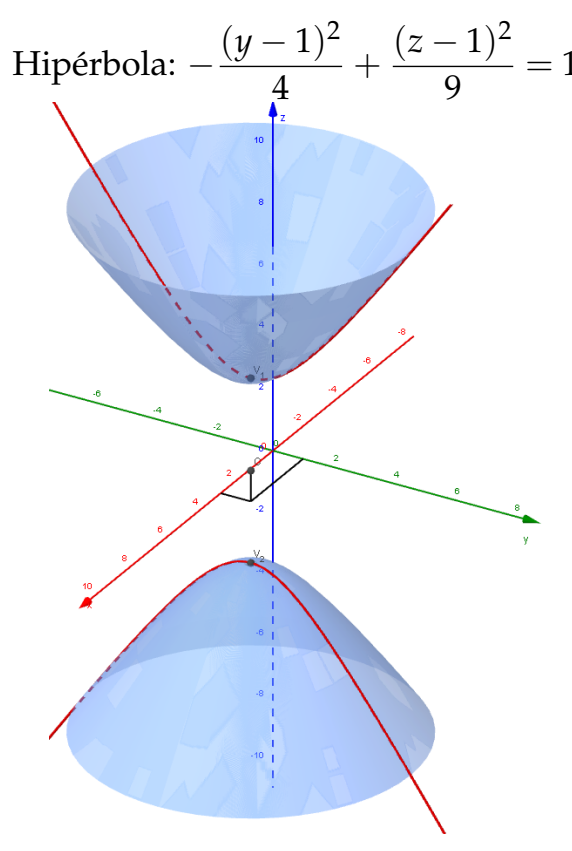

Traza $z=7$

Elipse: $\frac{(x-3)^{2}}{12}+\frac{(y-1)^{2}}{12}=1$

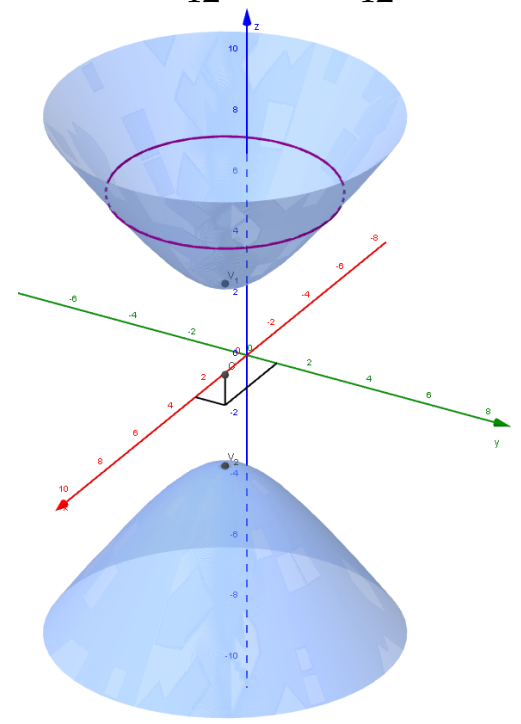

Traza $y=1$

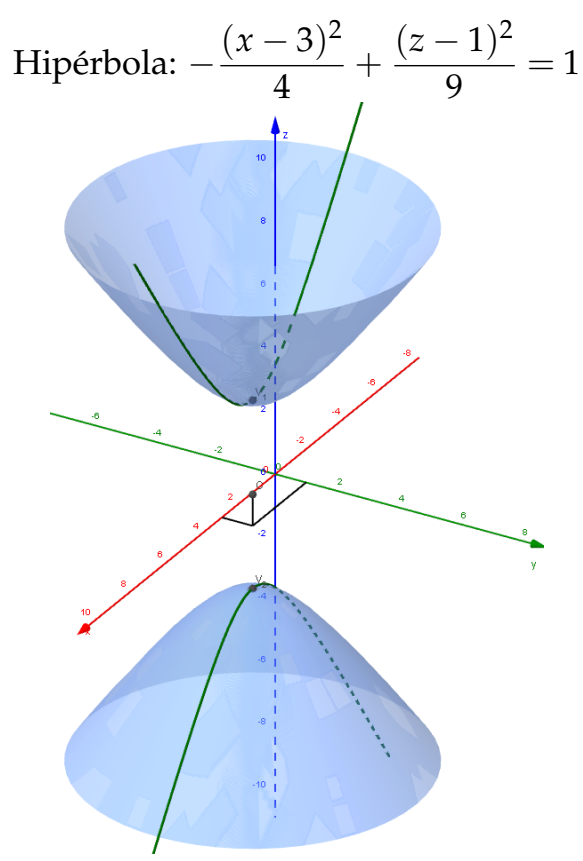

Traza $z=-5$

Elipse: $\frac{(x-3)^{2}}{12}+\frac{(y-1)^{2}}{12}=1$

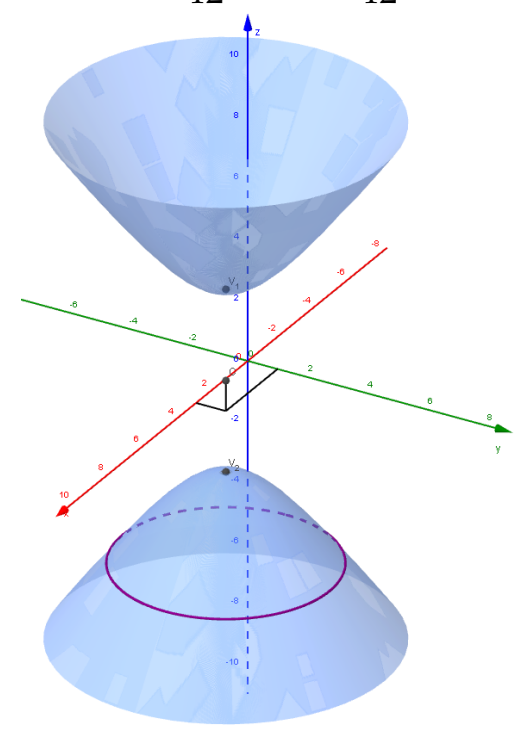

Nota: Recuerde que las trazas son solo las curvas representadas en rojo, verde y morado. 


\section{Applet para las trazas de un hiperboloide de dos hojas}

El siguiente applet, se pueden manipular de igual manera que el anterior.

Para ingresar al applet, debe dar click sobre la siguiente imagen o ingresar en el link: Applet Hiperboloide de dos hojas.
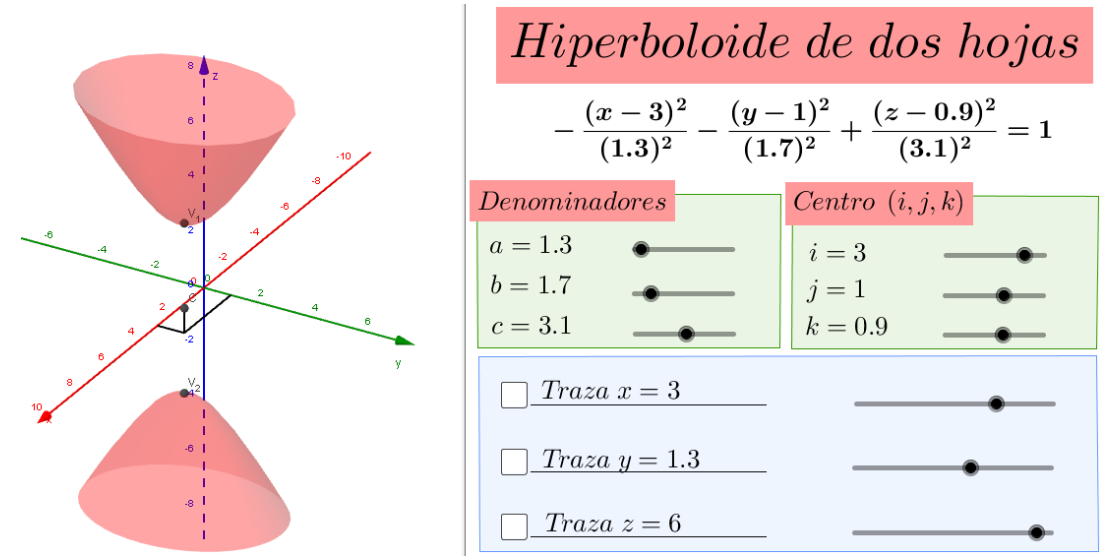

\section{Actividad}

Considere la superficie cuádrica $S$ de ecuación:

$$
-\frac{(x-2)^{2}}{4}-\frac{(y-2)^{2}}{2}+(z+1)^{2}=1
$$

Calcule y dibuje las trazas que se obtienen con los planos $x=2, y=2, z=2$ y $z=-4$.

\section{Actividad}

Considere la superficie cuádrica $H$ de ecuación:

$$
-(x+5)^{2}+\frac{y^{2}}{4}-\frac{(z+2)^{2}}{4}=1
$$

Calcule y dibuje las trazas que se obtienen con los planos $x=-5, y=4, y=-4$ y $z=-2$. 


\section{Cono elíptico}

\section{Definición 1.5}

La ecuación canónica del cono elíptico centrado en $C$, con eje en z es:

$$
\frac{(x-i)^{2}}{a^{2}}+\frac{(y-j)^{2}}{b^{2}}-\frac{(z-k)^{2}}{c^{2}}=0
$$

donde $C(i, j, k)$ es un punto en el espacio y $a, b, c$ son constantes reales positivas.

El eje del cono elíptico corresponde a la variable cuyo coeficiente es negativo.

\section{Ejemplo 1.7}

Considere el cono elíptico de ecuación:

$$
\frac{(x+1)^{2}}{4}+\frac{(y+2)^{2}}{1}-\frac{z^{2}}{9}=0
$$

A continuación se puede encontrar su representación gráfica:

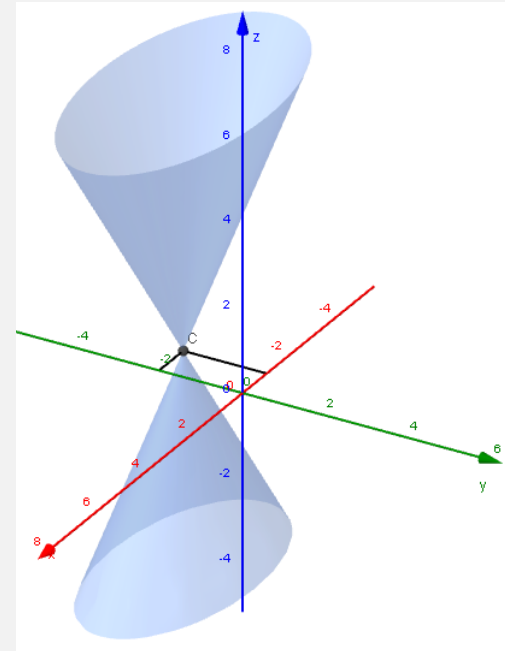

\section{Ejemplo 1.8}

Considere el cono elíptico de ecuación:

$$
\frac{(x+1)^{2}}{4}+\frac{(y+2)^{2}}{1}-\frac{z^{2}}{9}=0
$$

Calcule y dibuje las trazas que se obtienen con los planos $x=-1, y=-2, z=3$ y $z=-3$.

\section{Solución:}




$$
\text { Traza } x=-1
$$

Rectas: $3 y+-z+6=0$

$3 y+z+6=0$

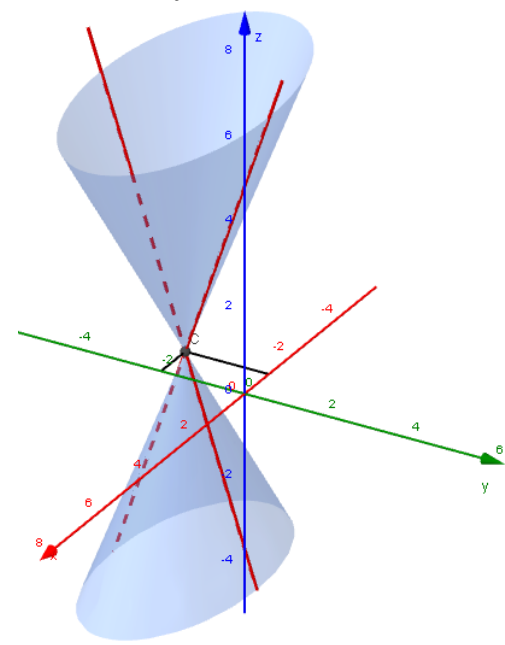

Traza $z=3$

Elipse: $\frac{(x+1)^{2}}{4}+\frac{(y+2)^{2}}{1}=1$

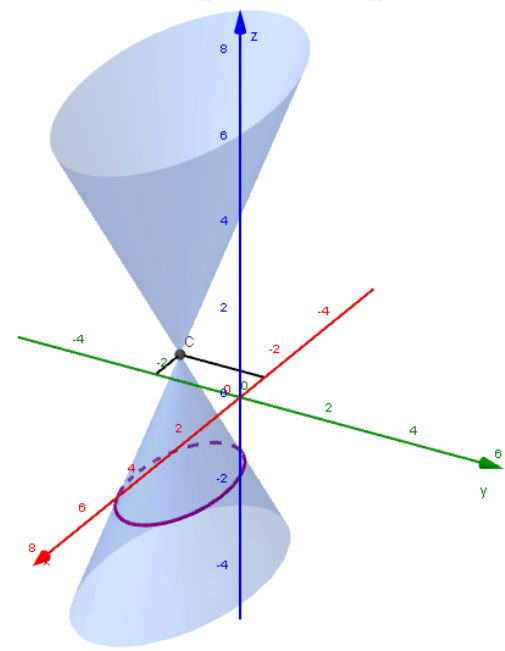

Traza $y=-2$

Rectas: $3 x-2 z+3=0$

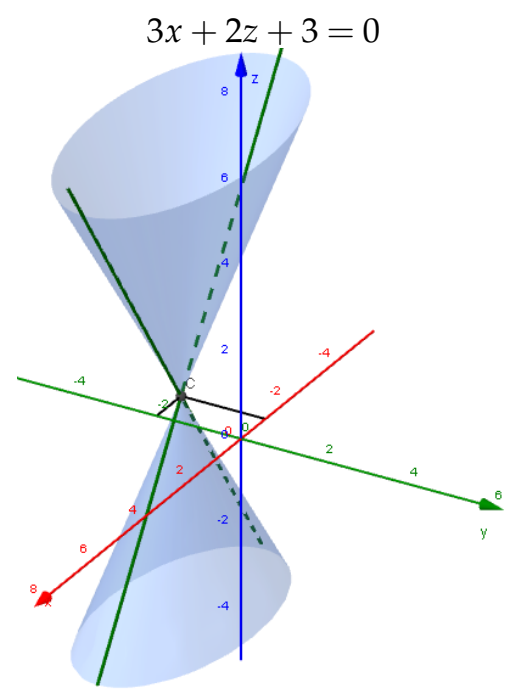

Traza $z=-3$

Elipse: $\frac{(x+1)^{2}}{4}+\frac{(y+2)^{2}}{1}=1$

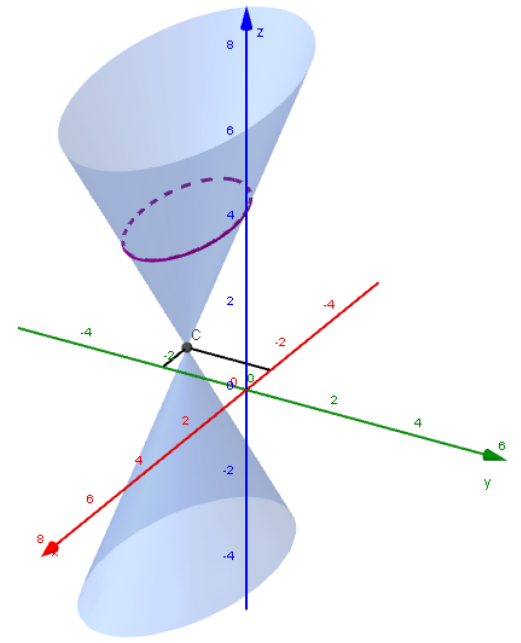

Nota: Recuerde que las trazas son solo las curvas representadas en rojo, verde y morado.

\section{Applet para las trazas de un cono elíptico}

El siguiente applet, se pueden manipular de igual manera que el anterior. 
Para ingresar al applet, debe dar click sobre la siguiente imagen o ingresar en el link: Applet Cono Elíptico.
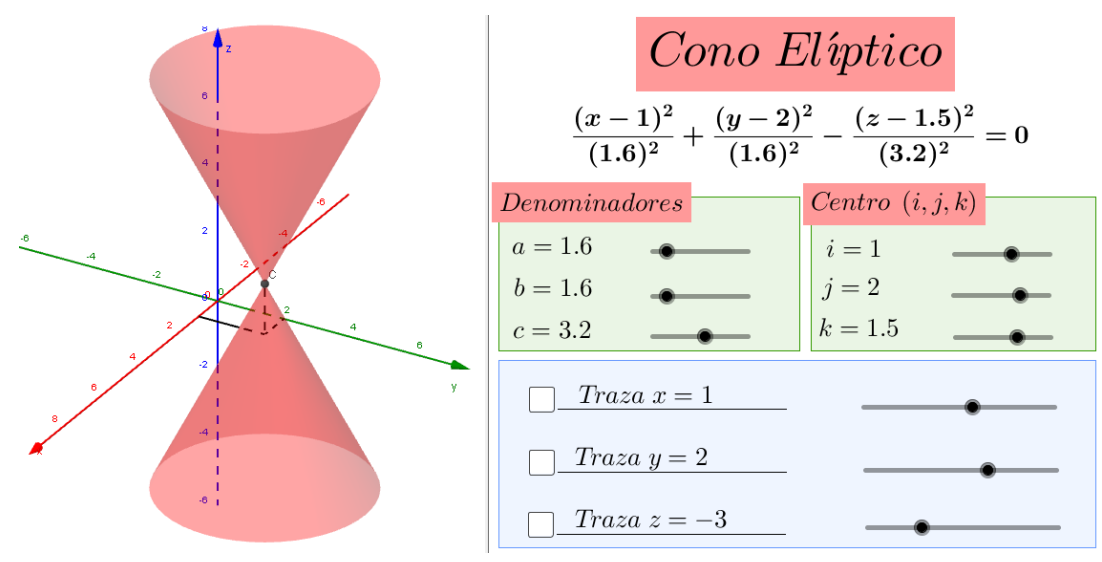

\section{Actividad}

Considere la superficie cuádrica $S$ de ecuación:

$$
\frac{(x-3)^{2}}{2}+\frac{(y+1)^{2}}{2}-\frac{(z-1)^{2}}{3}=0
$$

Calcule y dibuje las trazas que se obtienen con los planos $x=3, y=-1, z=4$ y $z=-2$.

\section{Actividad}

Considere la superficie cuádrica $C$ de ecuación:

$$
-\frac{(x+5)^{2}}{4}+\frac{y^{2}}{2}+\frac{(z+2)^{2}}{4}=0
$$

Calcule y dibuje las trazas que se obtienen con los planos $x=-3, x=-7, y=0$ y $z=-2$.

\section{Paraboloide elíptico}

\section{Definición 1.6}

La ecuación canónica del paraboloide elíptico con vértice en $C$, y eje en z es:

$$
z-k=\frac{(x-i)^{2}}{a^{2}}+\frac{(y-j)^{2}}{b^{2}}
$$


donde $C(i, j, k)$ es un punto en el espacio y $a, b$ son constantes reales positivas.

El eje del paraboloide elíptico corresponde a la variable lineal.

\section{Ejemplo 1.9}

Considere el paraboloide elíptico de ecuación:

$$
z-2=\frac{(x-1)^{2}}{4}+\frac{(y+1)^{2}}{4}
$$

A continuación se puede encontrar su representación gráfica:

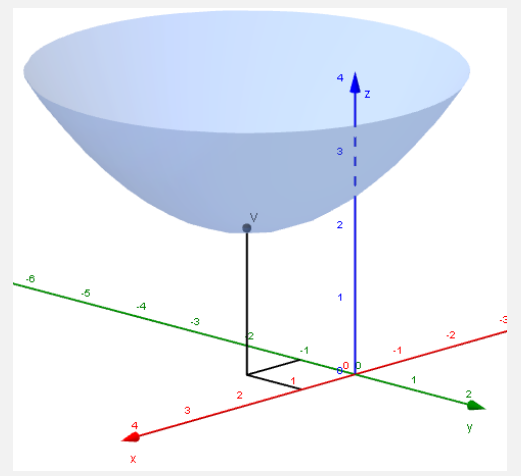

Ejemplo 1.10

Considere el paraboloide elíptico de ecuación:

$$
z-2=\frac{(x-1)^{2}}{4}+\frac{(y+1)^{2}}{4}
$$

Calcule y dibuje las trazas que se obtienen con los planos $x=1, y=-1$ y $z=4$.

\section{Solución:}




$$
\begin{array}{lll}
\text { Traza } x=1 \quad \text { Traza } y=-1 & \text { Traza } z=4
\end{array}
$$

Parábola: $z-2=\frac{(y+1)^{2}}{4}$

Parábola: $z-2=\frac{(x-1)^{2}}{4}$

Elipse: $\frac{(x-1)^{2}}{8}+\frac{(y+1)^{2}}{8}=1$
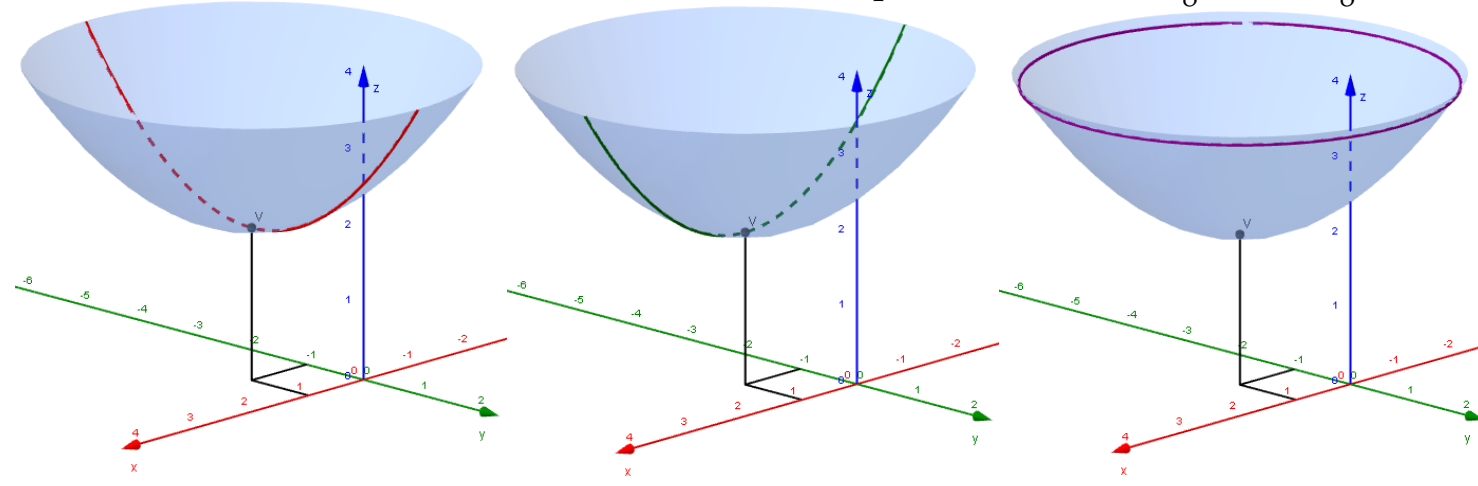

Nota: Recuerde que las trazas son solo las curvas representadas en rojo, verde y morado.

\section{Applet para las trazas de un paraboloide elíptico}

El siguiente applet, se pueden manipular de igual manera que el anterior.

Para ingresar al applet, debe dar click sobre la siguiente imagen o ingresar en el link: Applet Paraboloide Elíptico.

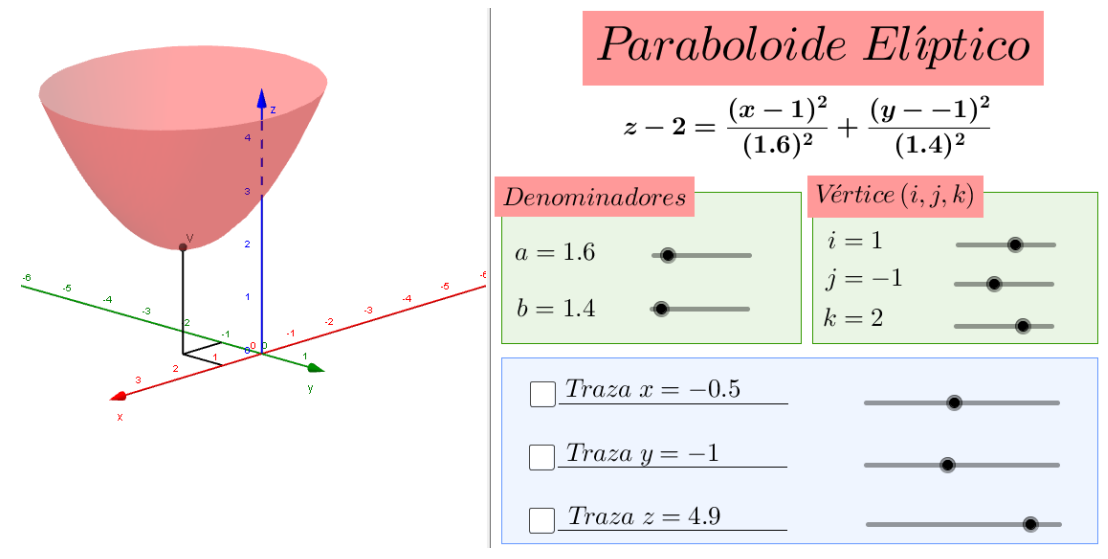

\section{Actividad}

Considere la superficie cuádrica $S$ de ecuación:

$$
z=\frac{(x-3)^{2}}{2}+\frac{(y+1)^{2}}{2}
$$


Calcule y dibuje las trazas que se obtienen con los planos $x=3, y=-1$ y $z=2$.

\section{Actividad}

Considere la superficie cuádrica $P$ de ecuación:

$$
2-y=\frac{(x+5)^{2}}{2}+\frac{(z+2)^{2}}{1}
$$

Calcule y dibuje las trazas que se obtienen con los planos $x=-5, y=0$ y $z=-2$.

\section{Paraboloide hiperbólico}

\section{Definición 1.7}

La ecuación canónica del paraboloide hiperbólico con centro en $C$, y eje en z es:

$$
z-k=-\frac{(x-i)^{2}}{a^{2}}+\frac{(y-j)^{2}}{b^{2}}
$$

donde $C(i, j, k)$ es un punto en el espacio y $a, b$ son constantes reales positivas.

El eje del paraboloide hiperbólico corresponde a la variable lineal.

\section{Ejemplo 1.11}

Considere el paraboloide hiperbólico de ecuación:

$$
z+1=-\frac{(x-1)^{2}}{9 / 4}+\frac{(y-2)^{2}}{9 / 4}
$$

A continuación se puede encontrar su representación gráfica:

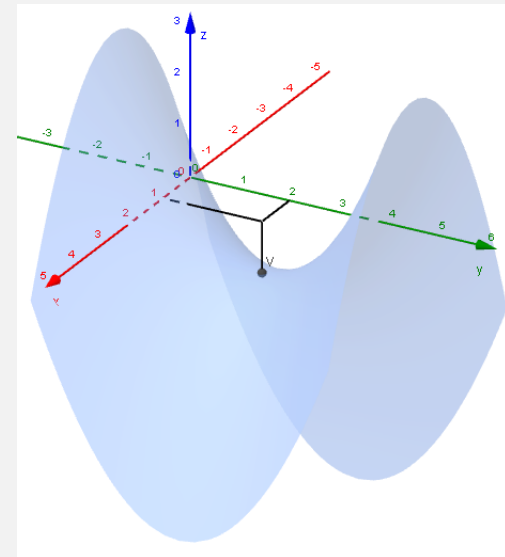


Ejemplo 1.12

Considere el paraboloide hiperbólico de ecuación:

$$
z+1=-\frac{(x-1)^{2}}{9 / 4}+\frac{(y-2)^{2}}{9 / 4}
$$

Calcule y dibuje las trazas que se obtienen con los planos $x=1, y=2$ y $z=-1$.

\section{Solución:}

$$
\text { Traza } x=1
$$

Parábola: $z+1=\frac{(y-2)^{2}}{9 / 4}$

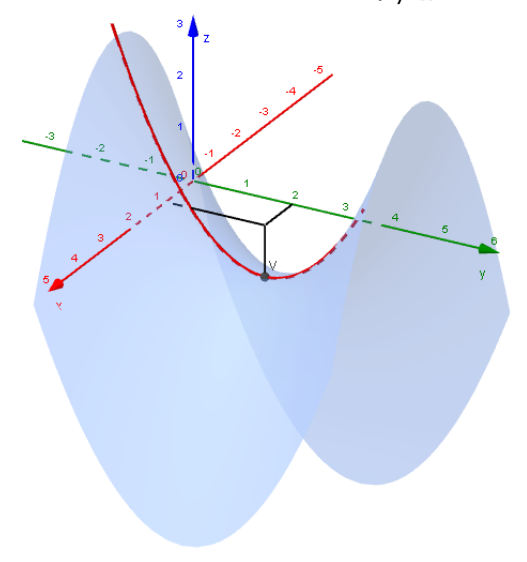

Traza $y=2$

Parábola: $z+1=-\frac{(x-1)^{2}}{9 / 4}$

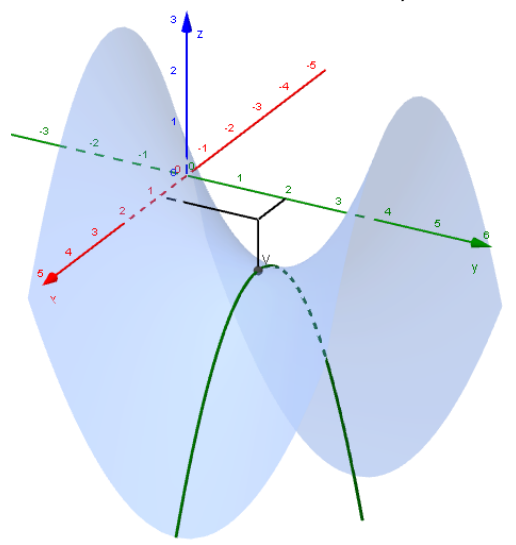

$\operatorname{Traza} z=-1$

Rectas: $y-x-1=0$

$y+x-3=0$

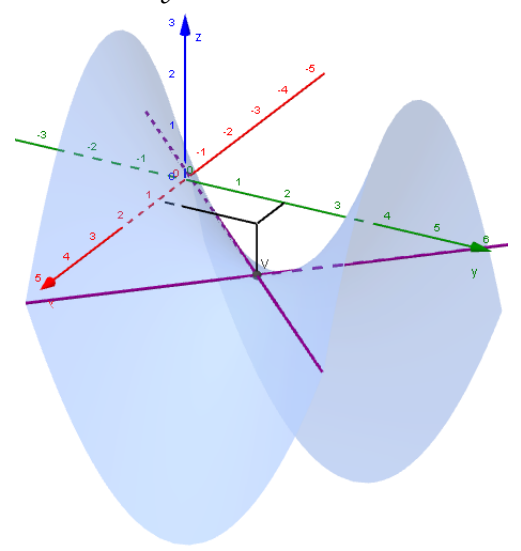

Nota: Recuerde que las trazas son solo las curvas representadas en rojo, verde y morado.

\section{Applet para las trazas de un paraboloide hiperbólico}

El siguiente applet, se pueden manipular de igual manera que el anterior.

Para ingresar al applet, debe dar click sobre la siguiente imagen o ingresar en el link: Applet Paraboloide Hiperbólico. 

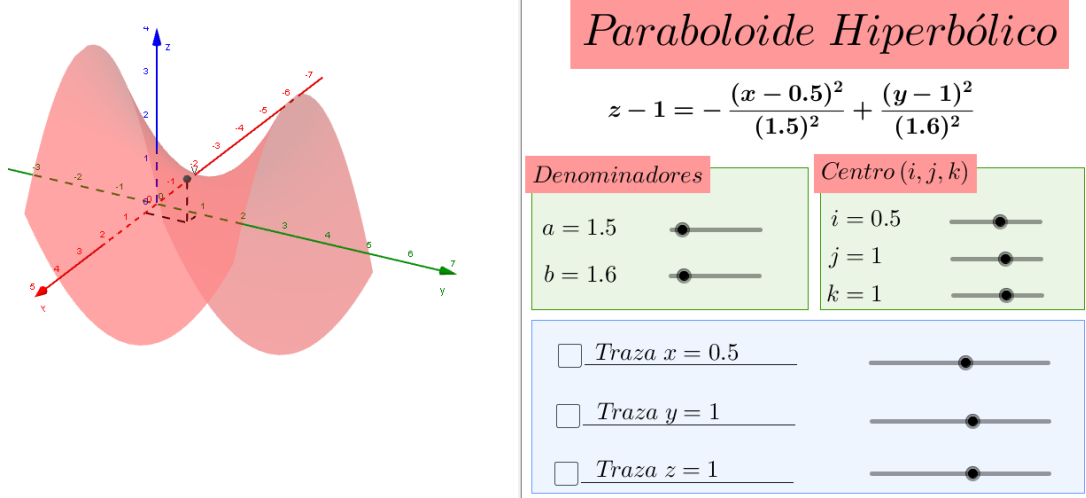

\section{Actividad}

Considere la superficie cuádrica $S$ de ecuación:

$$
z+1=\frac{(x-3)^{2}}{2}-\frac{(y+1)^{2}}{2}
$$

Calcule y dibuje las trazas que se obtienen con los planos $x=3, y=-1$ y $z=-1$.

\section{Actividad}

Considere la superficie cuádrica $P$ de ecuación:

$$
1-y=\frac{(x+1)^{2}}{4}-\frac{(z+2)^{2}}{1}
$$

Calcule y dibuje las trazas que se obtienen con los planos $x=-1, y=1$ y $z=-2$.

\subsection{Implementación de los Applets}

\section{Aspectos básicos de GeoGebra}

En esta sección se pretende mostrar al lector como se utilizó el programa GeoGebra para crear los applets utilizados anteriormente.

En primer lugar debe tener instalado en su computadora la herramienta GeoGebra, la cual es gratuita y se puede descargar en la siguiente dirección: https://www.geogebra.org/download. Cuando la tenga instalada, en sus programas aparecerá uno con el siguiente ícono: 


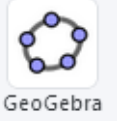

Al entrar al programa tendrá la siguiente pantalla de inicio:

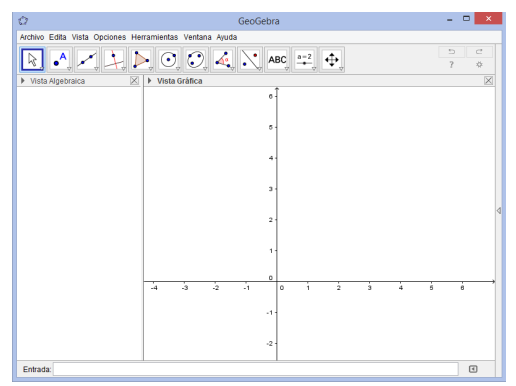

En el menú superior, ingrese a Vista y luego a Vista Gráfica 3D:

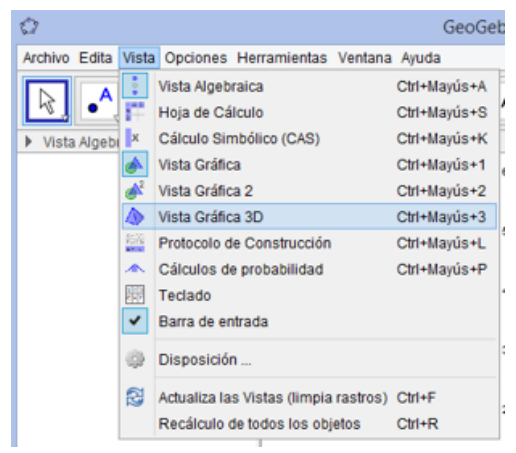

Maximice su ventana y ordene las vistas tal como se muestra a continuación:

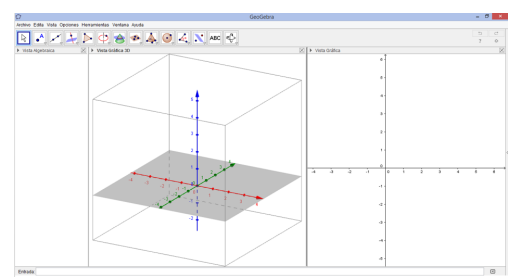

El menu en la parte superior cambiará de acuerdo a la vista gáfica que tenga seleccionada. En Vista Gráfica 3D, se tiene el siguiente menu:

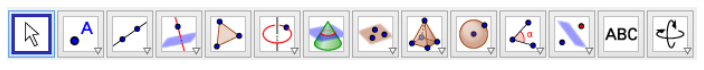

Y en la Vista Gráfica 2D, se tiene el menu:

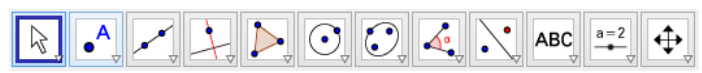

De click derecho sobre la Vista Gráfica 3D y desmarque la opcion de plano. 


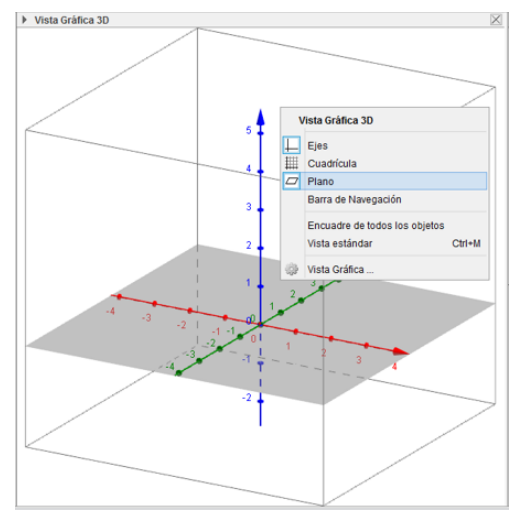

Nuevamente, con click derecho sobre la Vista Gráfica 3D ingrese a Vista Gráfica para poder modificar algunas preferencias. En preferencias desmarque Mostrar el recorte y cambie el tamaño de la caja a grande.

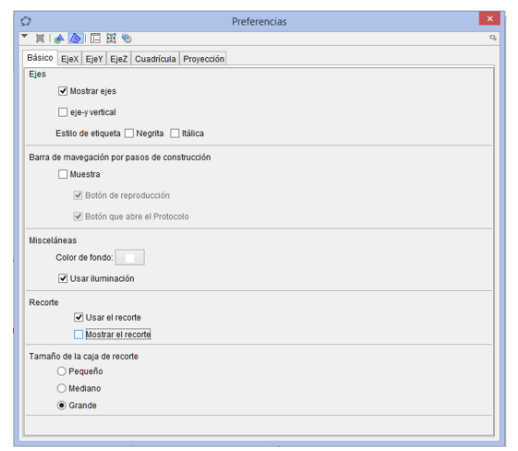

En preferencias hay otro menú en la parte superior, seleccione EjeX para rotular el eje, en Rótulo escoja la $x$. Realice lo mismo para los ejes y y $z$, y cierre la ventana.

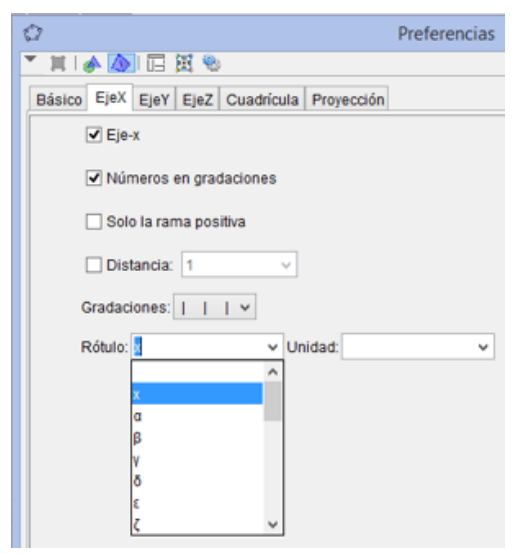

La Vista Gráfica 3D, se puede girar, para logarlo seleccione el ícono programa.

Luego de click sobre la Vista Gráfica 3D y manteniendo presionado el ratón, deslice hacia arriba, abajo, derecha o izquierda el sistema de coordenadas para girarlo. 


\section{Graficando la superficie}

Para poder graficar una superficie en GeoGebra, primero se tiene que parametrizar.

En el caso del Elipsoide, con ecuación canónica:

$$
\frac{(x-i)^{2}}{a^{2}}+\frac{(y-j)^{2}}{b^{2}}+\frac{(z-k)^{2}}{c^{2}}=1
$$

Su parametrización estaría dada por:

$$
s(u, v):\left\{\begin{array}{l}
x=i-a \cos u \operatorname{sen} v \\
y=j-b \operatorname{sen} u \operatorname{sen} v ; \quad u \in[0,2 \pi], v \in[0, \pi] \\
z=k-c \cos v
\end{array}\right.
$$

\section{Ejemplo 1.13}

Considere el elipsoide de ecuación:

$$
\frac{(x-1)^{2}}{4}+\frac{(y-2)^{2}}{9}+\frac{(z-2)^{2}}{1}=1
$$

Realice la gráfica de la superficie en GeoGebra.

\section{Solución:}

Primero se parametriza

$$
s(u, v):\left\{\begin{array}{l}
x=1-2 \cos u \operatorname{sen} v \\
y=2-3 \operatorname{sen} u \operatorname{sen} v ; \quad u \in[0,2 \pi], v \in[0, \pi] \\
z=2-1 \cos v
\end{array}\right.
$$

Ahora se ingresa a GeoGebra la superficie, para esto se escribe la palabra "Superficie " en la celda de entrada que se encuentra en la parte inferior de la pantalla, GeoGebra le completará la guía para ingresar la superficie, usted debe seleccionarla:

Recuede que esta superficie está parametrizada, y depede de 2 parámetros.

Donde dice $<$ Expresión $>$ se debe escribir la parametrización de las tres variables, en el orden $x, y, z$. En $<$ Parámetro $1>$ se escribe $u,<$ Valor inicial $1>$ y $<$ Valor final $1>$ corresponde a los extremos del intervalo donde se evaluará el parámetro 1 , en este caso $u \in[0,2 \pi]$.

En $<$ Parámetro $2>$ se escribe $v,<$ Valor inicial $2>$ y $<$ Valor final $2>$ corresponde a los extremos del intervalo donde se evaluará el parámetro 2 , en este caso $v \in[0, \pi]$.

Su entrada debe quedar de la siguiente manera:

Entrada: Superficie[1- $\left.2^{\star} \cos (u)^{\star} \operatorname{sen}(\mathrm{V}), 2-3^{\star} \operatorname{sen}(u)^{\star} \operatorname{sen}(\mathrm{V}), 2-1^{\star} \cos (\mathrm{V}), \mathrm{u}, \mathbf{0}, \mathbf{2}^{\star} \mathrm{pi}, \mathrm{v}, \mathbf{0}, \mathrm{pi}\right]$ 
El valor de $\pi$ lo puede escribir como pi, o tomar al final de la línea donde está escribiendo, dando click en: $₫$.

Cuando halla terminado, pulse la tecla "Enter " para poder ver su superficice. Puede girarla para tener diferentes vistas.
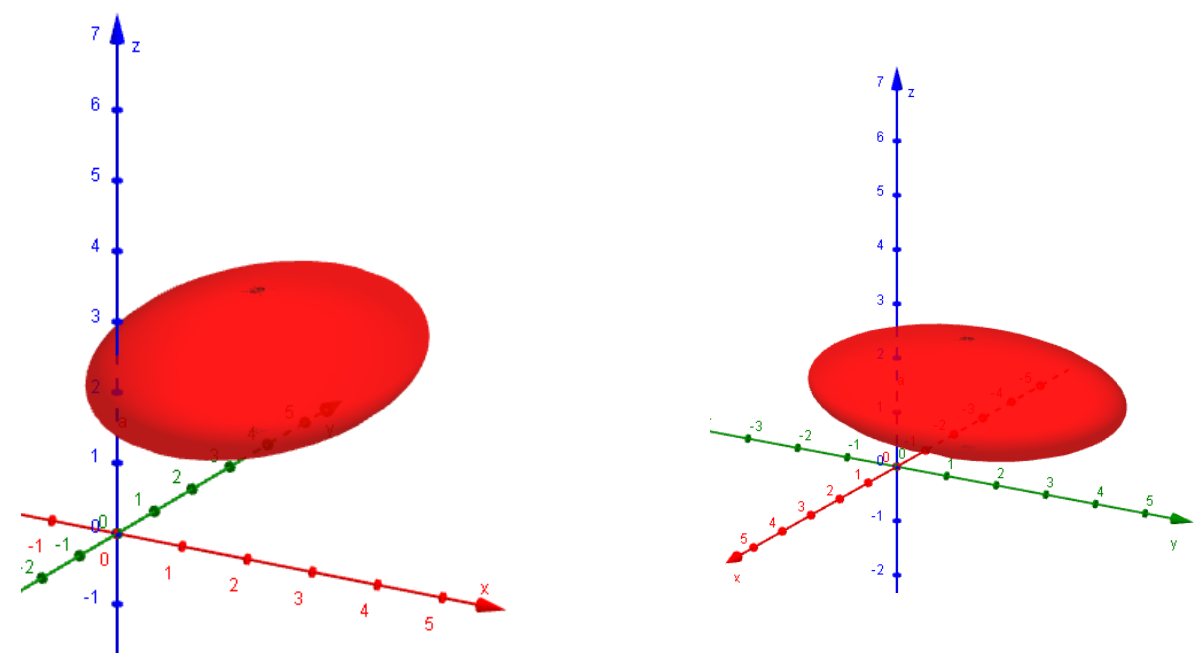

Cada vez que se crea un objeto utilizando la celda de entrada, debe terminar pulsando la tecla "Enter " para que el programa grafique el objeto editado.

La superficie que acabamos de graficar, es una superficie fija, por que sus valores para $a, b, c, i, j, k$ no varían.

Ahora se hará una superficie donde estos valores cambien, para esto hay que crear algunos deslizadores.

De click derecho sobre la Vista Gráfica 2D y desmarque la opción de ejes.

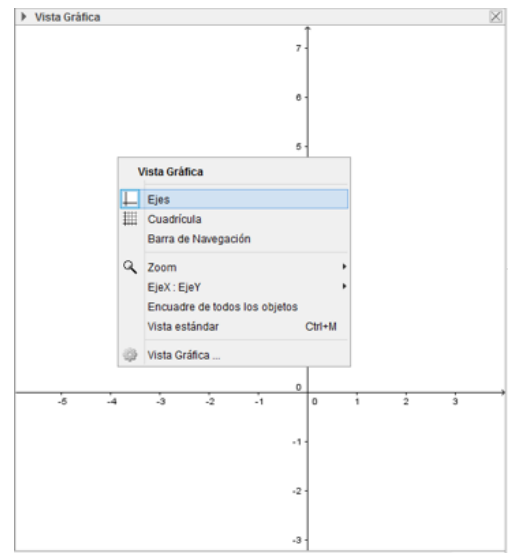

Seleccione el deslizador del menu superior:

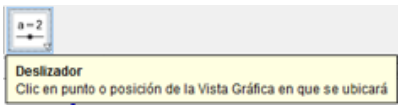


De click en la Vista Gráfica 2D donde desea colocar el deslizador, y complete la información que se le solicita, el deslizador se llamará $a$, con un valor mínimo de 1 y máximo de 5, luego seleccione Aplicar.

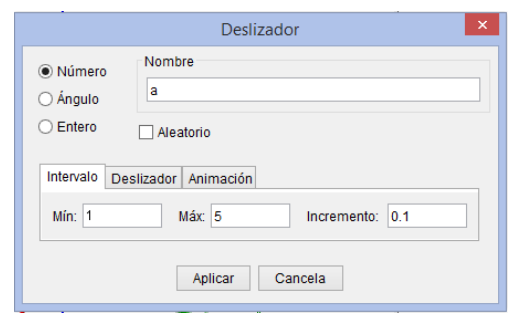

Debe crear dos deslizadores más $b$ y $c$ con las mismas características.

Si desea cambiar alguna de las propiedades del deslizador, solo debe dar click derecho sobre la línea del deslizador y seleccionar propiedades:

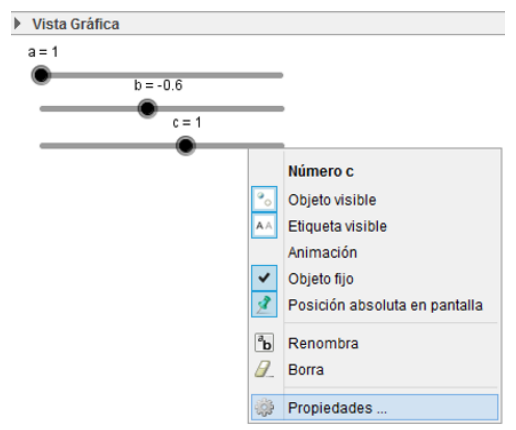

Para crear los deslizadores del centro del elipsoide, use la misma herramienta, lo que cambiará son las características. Se necesitan tres más, $i, j$ y $k$, con un valor mínimo de -5 y máximo de 5 , luego seleccione Aplicar.

Por el momento debería tener los siguientes deslizadores:

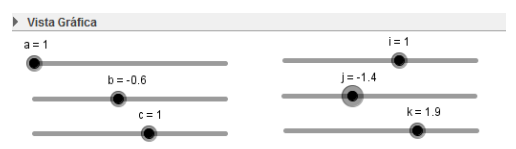

Para ocualtar o mostrar los objetos creados, puede utilizar la Vista Algebraica, debe dar click en el punto azul que aparece al lado izquierdo de cada objeto, si el punto está en blanco el objeto está oculto. Oculte la primer superficie creada.

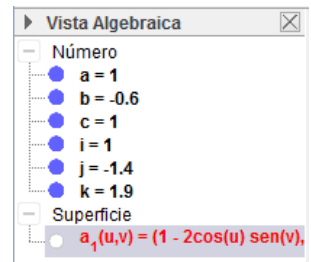

Ahora se creará un elipsoide que dependa de los deslizadores creados anteriormente, escriba la palabra "superficie" en la celda de entrada que se encuentra en la parte inferior de la pantalla, y complete con la sugerencia que le muestra el programa: 
Cambie las entradas de la superficie de la siguiente manera:

$$
\text { Entrada: Superficie[i - a } \left.\cos (u) \operatorname{sen}(v), j-b \operatorname{sen}(u) \operatorname{sen}(v), k-c \cos (v), u, 0,2^{\star} p i, v, 0, p i\right]
$$

Lo que se muestra en azul, son los deslizadores creados anteriormente, y lo que aparece en gris son los parametros de la superficie.

Cuando halla terminado, pulse la tecla "Enter" para poder ver su superficice. Puede girarla para tener diferentes vistas, y variar los deslizadores para obtener diferentes superficies.
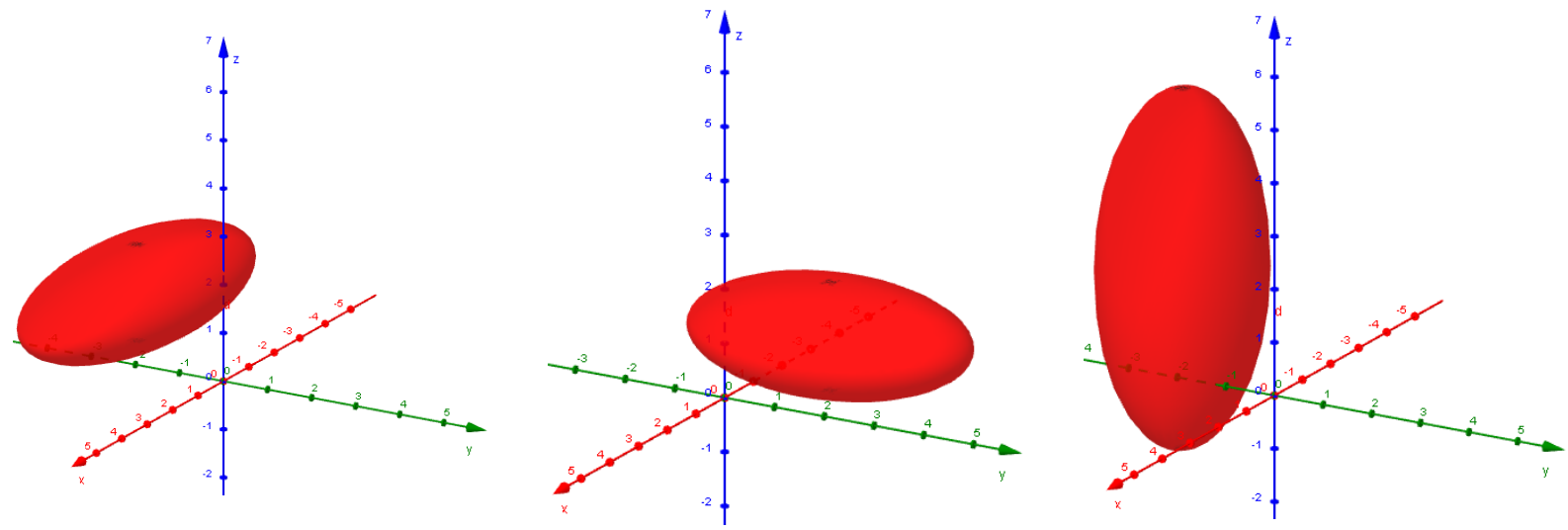

Para graficar el centro de la superficie, y que éste varíe conforme mueve los deslizadores, escriba en la celda de entrada: $(i, j, k)$ y pulse la tecla "Enter", con esto se creará un punto el cual se mueve cada vez que varíe el deslizador:

Entrada: (i,j,k)

\section{Graficando las trazas}

Las trazas también se van a hacer dependiendo de un deslizador, haga un deslizador que se llame $\operatorname{Traza}_{x}$, con un valor mínimo de -7 y máximo de 7 , luego seleccione Aplicar.

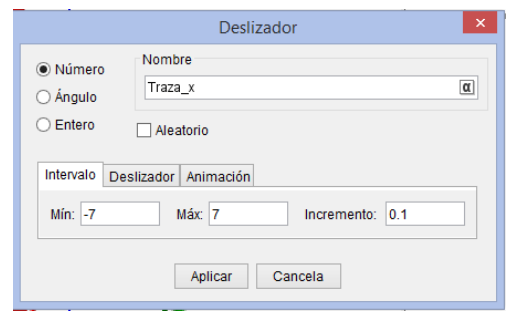

Debe crear dos deslizadores más Trazay y Trazaz con las mismas características.

Haga tres vectores, escriba en la celda de entrada: Vector $(1,0,0)$, y pulse la tecla "Enter ". Agregue los otros 2 vectores, que serían $\operatorname{Vector}(0,1,0)$ y $\operatorname{Vector}(0,0,1)$, de la misma manera. 


\section{Traza en $\mathrm{x}$}

Para hacer el plano, escriba en la celda de entrada "plano ", de las opciones que le presenta GeoGebra escoja la última: "PlanoPerpendicular $[<$ Punto $>,<$ Vector $>$ ]" y complétela de la siguiente manera:

Entrada: PlanoPerpendicular[(Traza_x,0,0), u]

Ahora, mueva el parámetro y vea como se mueve el plano y como corta al elipsoide.
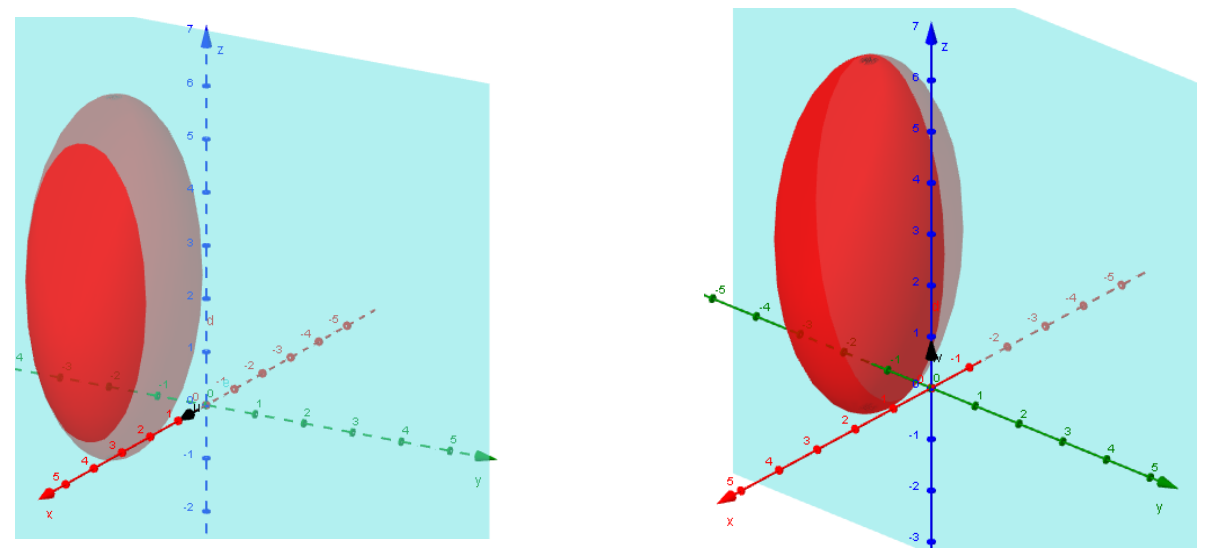

Se calculará la curva de intersección de manera algebraica, para luego poder graficarla.

$$
\begin{aligned}
& \frac{(x-i)^{2}}{a^{2}}+\frac{(y-j)^{2}}{b^{2}}+\frac{(z-k)^{2}}{c^{2}}=1 \quad \cap \quad x=\operatorname{Tr}^{2} a a_{x} \\
& \frac{\left(\operatorname{Tr} a z a_{x}-i\right)^{2}}{a^{2}}+\frac{(y-j)^{2}}{b^{2}}+\frac{(z-k)^{2}}{c^{2}}=1 \\
& \frac{(y-j)^{2}}{b^{2}}+\frac{(z-k)^{2}}{c^{2}}=1-\frac{\left(\operatorname{Tr} a z a_{x}-i\right)^{2}}{a^{2}} \\
& \frac{(y-j)^{2}}{b^{2}}+\frac{(z-k)^{2}}{c^{2}}=\frac{a^{2}-\left(\operatorname{Tr} a z a_{x}-i\right)^{2}}{a^{2}} \\
& \frac{(y-j)^{2}}{b^{2} \frac{a^{2}-\left(\operatorname{Traza} a_{x}-i\right)^{2}}{a^{2}}}+\frac{(z-k)^{2}}{c^{2} \frac{a^{2}-\left(\operatorname{Tr} a z a_{x}-i\right)^{2}}{a^{2}}}=1 \\
& \frac{(y-j)^{2}}{\left(\frac{b}{a} \sqrt{a^{2}-\left(\operatorname{Tr} a z a_{x}-i\right)^{2}}\right)^{2}}+\frac{(z-k)^{2}}{\left(\frac{c}{a} \sqrt{a^{2}-\left(\operatorname{Tr} a z a_{x}-i\right)^{2}}\right)^{2}}=1
\end{aligned}
$$

Con lo anterior se obtiene la ecuación de una elipse, y para graficarla en GeoGebra, se debe parametrizar.

En el caso de la Elipse, con ecuación canónica:

$$
\frac{(x-i)^{2}}{a^{2}}+\frac{(y-j)^{2}}{b^{2}}=1
$$


Su parametrización estaría dada por:

$$
c(t):\left\{\begin{array}{l}
x=i+a \cos t \\
y=j+b \operatorname{sen} t
\end{array} ; \quad t \in[0,2 \pi]\right.
$$

Esta curva está en el plano $z=0$.

Ahora, se debe adaptar de manera similar para la curva que resultó de la intersección del elipsoide y del plano:

$$
c(t):\left\{\begin{array}{l}
x=\operatorname{Traza} a_{x} \\
y=j+\left(\frac{b}{a} \sqrt{a^{2}-\left(\operatorname{Traza} a_{x}-i\right)^{2}}\right) \cos t ; \\
z=k+\left(\frac{c}{a} \sqrt{a^{2}-\left(\operatorname{Traza} a_{x}-i\right)^{2}}\right) \operatorname{sen} t
\end{array} \quad t \in[0,2 \pi]\right.
$$

Para hacer la curva, escriba la palabra "Curva" en la celda de entrada y de las opciones que le presenta GeoGebra escoja la segunda: "Curva[ <Expresión $>$, $<$ Expresión $>$, < Expresión $>$, $<$ Parámetro $>$, $<$ Valor inicial $>,<$ Valor final $>$ ]" que es la curva en 3D y complétela de la siguiente manera:

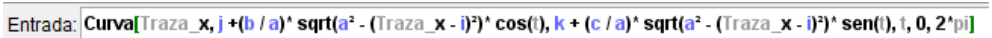

Ahora, mueva el parámetro y vea como se mueve el plano y la curva.
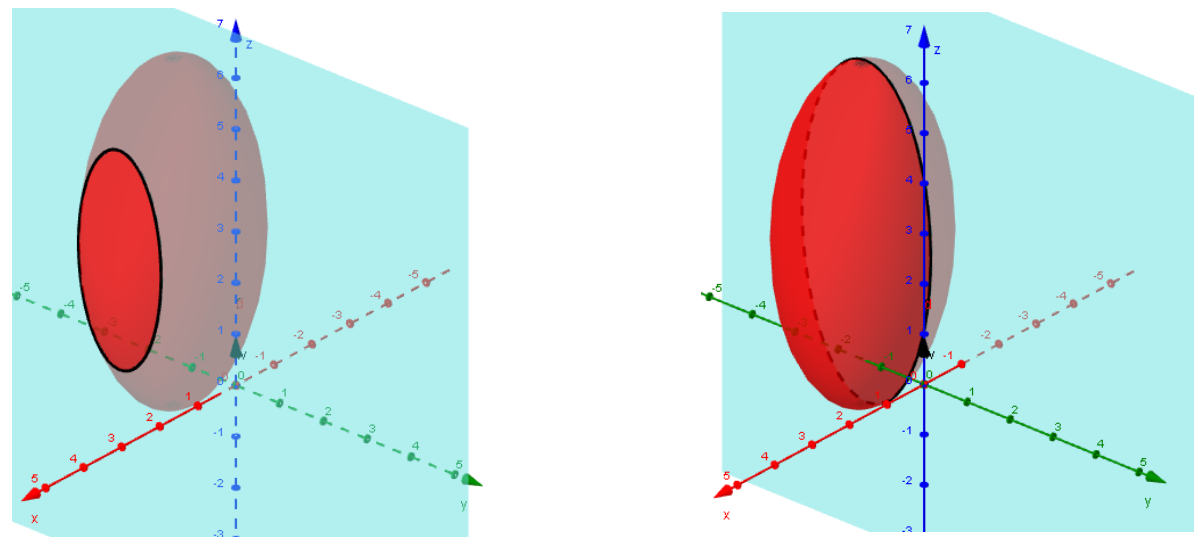

Recuerde que para ocultar o mostrar los objetos creados, puede utilizar la Vista Algebraica, para ello debe dar click en el punto azul que aparece al lado izquierdo de cada objeto, si el punto está en blanco el objeto está oculto. Con esto, puede ocultar el plano y la curva de la traza en $x$ para poder observar el plano y la curva de la traza en $y$.

\section{Traza en y}

Para hacer el plano, escriba en la celda de entrada: 
Se calculará la curva de intersección de manera algebraica, para luego poder graficarla.

$$
\begin{aligned}
& \frac{(x-i)^{2}}{a^{2}}+\frac{(y-j)^{2}}{b^{2}}+\frac{(z-k)^{2}}{c^{2}}=1 \cap y=\operatorname{Traza}_{y} \\
& \frac{(x-i)^{2}}{a^{2}}+\frac{\left(\operatorname{Tr}^{2} a a_{y}-j\right)^{2}}{b^{2}}+\frac{(z-k)^{2}}{c^{2}}=1 \\
& \frac{(x-i)^{2}}{a^{2}}+\frac{(z-k)^{2}}{c^{2}}=1-\frac{\left(\operatorname{Tr}^{2} a a_{y}-j\right)^{2}}{b^{2}} \\
& \frac{(x-i)^{2}}{a^{2}}+\frac{(z-k)^{2}}{c^{2}}=\frac{b^{2}-\left(\operatorname{Tr} a z a_{y}-j\right)^{2}}{b^{2}} \\
& \frac{(x-i)^{2}}{a^{2} \frac{b^{2}-\left(\operatorname{Traza} a_{y}-j\right)^{2}}{b^{2}}}+\frac{(z-k)^{2}}{c^{2} \frac{b^{2}-\left(\operatorname{Tr} a z a_{y}-j\right)^{2}}{b^{2}}}=1 \\
& \frac{(x-i)^{2}}{\left(\frac{a}{b} \sqrt{b^{2}-\left(T r a z a_{y}-j\right)^{2}}\right)^{2}}+\frac{(z-k)^{2}}{\left(\frac{c}{b} \sqrt{b^{2}-\left(\operatorname{Tr} a z a_{y}-j\right)^{2}}\right)^{2}}=1
\end{aligned}
$$

Con lo anterior se obtiene la ecuación de una elipse, y para graficarla en GeoGebra, se debe parametrizar.

$$
c(t):\left\{\begin{array}{l}
x=i+\left(\frac{a}{b} \sqrt{b^{2}-\left(\operatorname{Traza}_{y}-j\right)^{2}}\right) \cos t \\
y=\operatorname{Traza} a_{y} \\
z=k+\left(\frac{c}{b} \sqrt{b^{2}-\left(\operatorname{Tr} a a_{y}-j\right)^{2}}\right) \operatorname{sen} t
\end{array} ; \quad t \in[0,2 \pi]\right.
$$

Para hacer la curva, escriba en la celda de entrada:

Entrada: Curva[i + (a / b b ${ }^{\star} \operatorname{sqrt}\left(b^{2}-(\text { Traza } y-j)^{2}\right)^{\star} \cos (t)$, Traza $\left.y, k+(c / b)^{\star} \operatorname{sqrt}\left(b^{2}-(\text { Traza } y-j)^{2}\right)^{\star} \operatorname{sen}(t), t, 0,2^{\star} p i\right]$

Ahora, mueva el parámetro y vea como se mueve el plano y la curva.
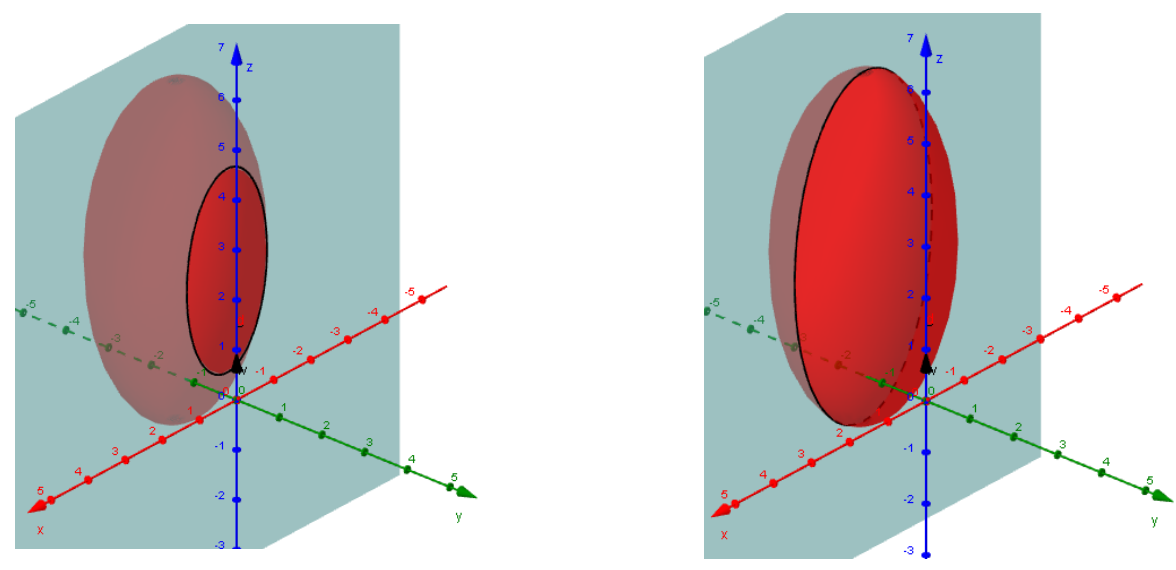


\section{Traza en z}

Para hacer el plano, escriba en la celda de entrada:

\section{Entrada: PlanoPerpendicular[(0,0,Traza_z), w]}

Se calculará la curva de intersección de manera algebraica, para luego poder graficarla.

$$
\begin{aligned}
& \frac{(x-i)^{2}}{a^{2}}+\frac{(y-j)^{2}}{b^{2}}+\frac{(z-k)^{2}}{c^{2}}=1 \quad \cap \quad z=\operatorname{Tr}^{2} a a_{z} \\
& \frac{(x-i)^{2}}{a^{2}}+\frac{(y-j)^{2}}{b^{2}}+\frac{\left(\operatorname{Traza}_{z}-k\right)^{2}}{c^{2}}=1 \\
& \frac{(x-i)^{2}}{a^{2}}+\frac{(y-j)^{2}}{b^{2}}=1-\frac{\left(\operatorname{Tr} a z a_{z}-k\right)^{2}}{c^{2}} \\
& \frac{(x-i)^{2}}{a^{2}}+\frac{(y-j)^{2}}{b^{2}}=\frac{c^{2}-\left(T r a z a_{z}-k\right)^{2}}{c^{2}} \\
& \frac{(x-i)^{2}}{a^{2} \frac{c^{2}-\left(\operatorname{Traza} a_{z}-k\right)^{2}}{c^{2}}}+\frac{(y-j)^{2}}{b^{2} \frac{c^{2}-(\operatorname{Traza}-k)^{2}}{c^{2}}}=1 \\
& \frac{(x-i)^{2}}{\left(\frac{a}{c} \sqrt{c^{2}-\left(\operatorname{Tr} a z a_{z}-k\right)^{2}}\right)^{2}}+\frac{(y-j)^{2}}{\left(\frac{b}{c} \sqrt{c^{2}-\left(\operatorname{Tr} a z a_{z}-k\right)^{2}}\right)^{2}}=1
\end{aligned}
$$

Con lo anterior se obtiene la ecuación de una elipse, y para graficarla en GeoGebra, se debe parametrizar.

$$
c(t):\left\{\begin{array}{l}
x=i+\left(\frac{a}{c} \sqrt{c^{2}-\left(\operatorname{Traza}_{z}-k\right)^{2}}\right) \cos t \\
y=j+\left(\frac{b}{c} \sqrt{c^{2}-\left(\operatorname{Traza} a_{z}-k\right)^{2}}\right) \operatorname{sen} t ; \quad t \in[0,2 \pi] \\
z=\operatorname{Traza}_{z}
\end{array}\right.
$$

Para hacer la curva, escriba en la celda de entrada: 

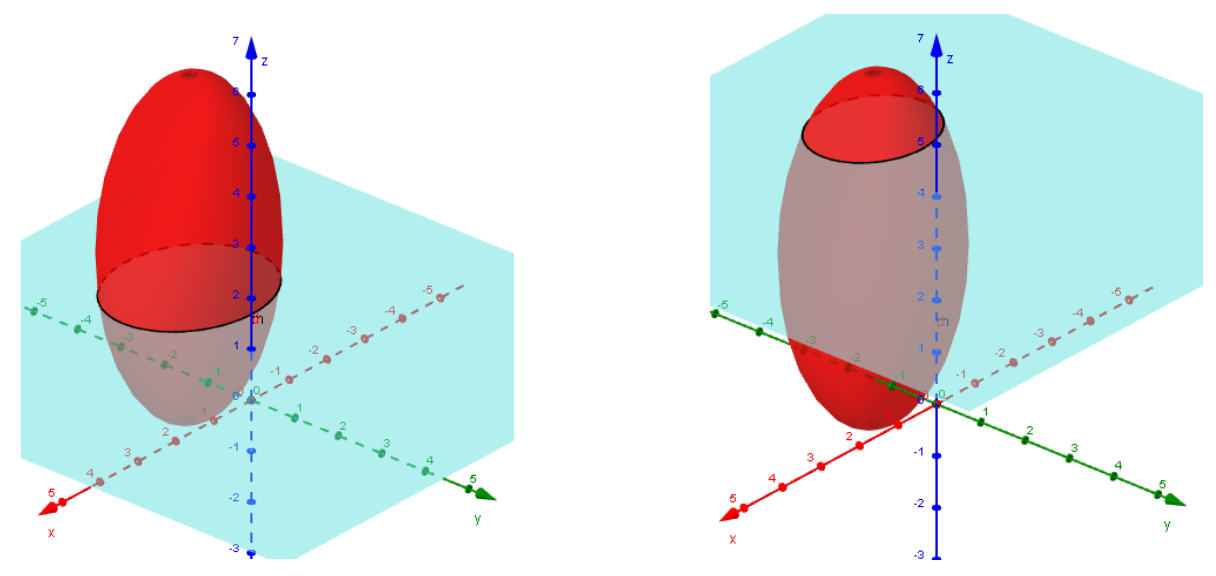

\section{Diseño y manipulación de objetos visibles}

Todos los objetos creados pueden ser modificados en color, grosor e inclusive en ser visibles o invisibles. Basta con dar click derecho sobre el objeto, en la Vista Gráfica 2D o 3D o sobre su nombre en la Vista Algebraica, y seleccionar Propiedades.

Puede probar cambiando el color de la superficie, planos y curvas. Y el grosor o estilo de línea en las curvas.

Para identificar mejor las curvas, cambie el nombre de éstas, la primera la puede llamar TrazaX, para esto entre a propiedades y cambie su nombre. Con las otras dos curvas puede hacer un cambio similar con TrazaY y TrazaZ.

De la misma manera cambie los nombres de los planos por: PlanoTrazaX, PlanoTrazaY y PlanoTrazaZ.

Para controlar si un objeto es visible o invisible, posicione el ratón sobre la Vista Gráfica 2D, y seleccione del menú superior la Casilla de control:

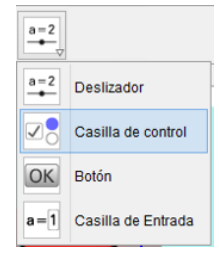

De click en la Vista Gráfica 2D donde desea colocar la casilla de control, y complete la información que se le solicita, la casilla se llamará Traza $x$, y en objetos debe seleccionar de la lista, los objetos que desee que sean visibles cuando la casilla de control este seleccionada. En este caso, seleccione: Curva paramétrica TrazaX y Plano PlanoTrazaX, luego seleccione Aplicar. 


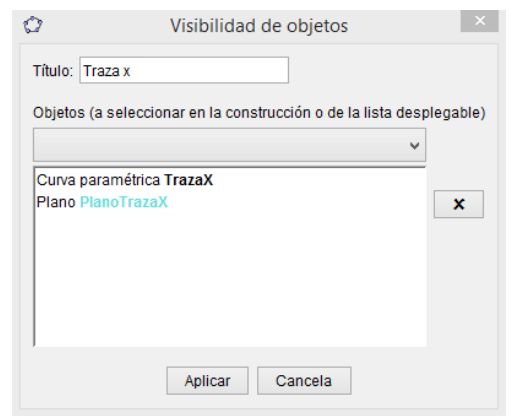

Debe crear dos Casillas de conrol más Traza y y Traza z para ocultar y mostrar el plano y la curva que corresponde a cada traza.

Puede probar marcando y desmarcando la casilla de control, para que observe como se muestra o no el plano y la curva de las trazas.

Ahora puede cerrar la Vista Algebraica, y ordenar a su gusto la Vista Gráfica 2D. Si desea escribir algún texto, posicione el ratón sobre la Vista Gráfica 2D, y seleccione del menú superior la herramienta de texto:

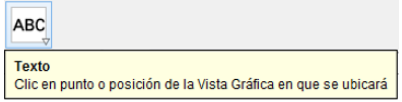

De click en la Vista Gráfica 2D donde desea colocar el texto, y complete la información que se le solicita:

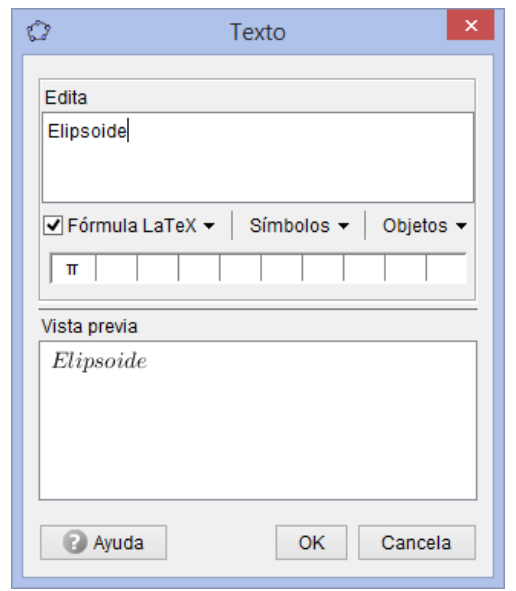

Para cambiar de tamaño y de color de fondo y texto, debe ingresar a las propiedades y modificarlo según su preferencia:
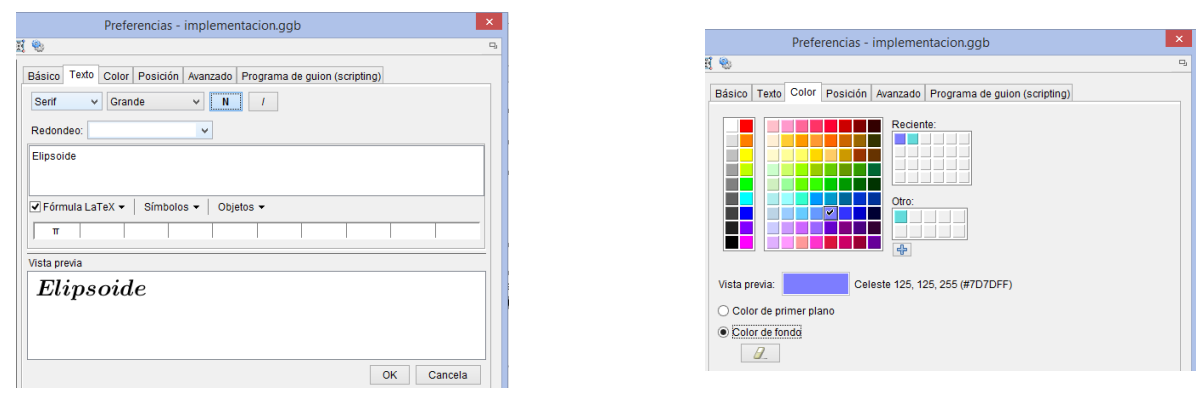
El trabajo realizado hasta este momento, debería verse similar a la siguiente imagen:

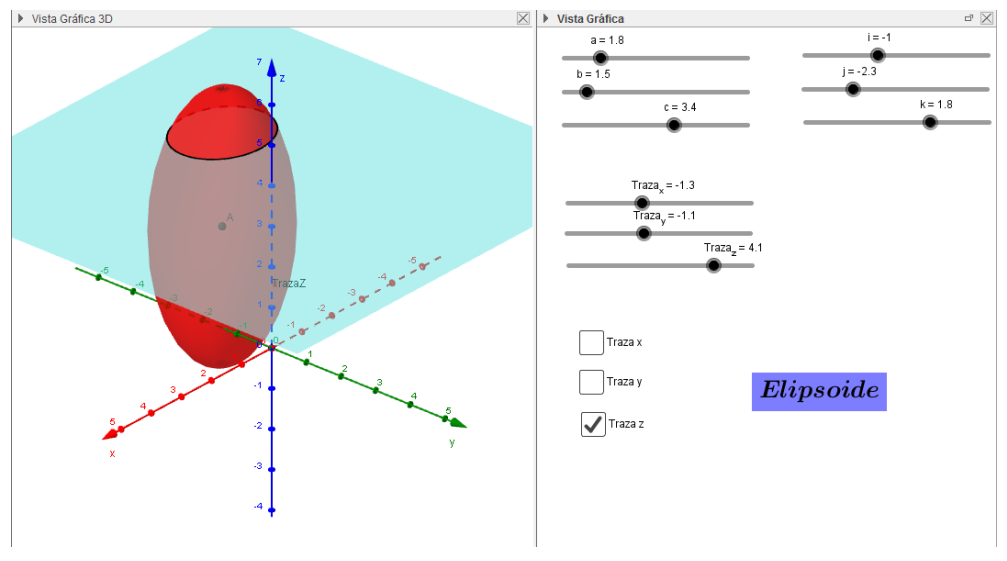

\subsection{Parametrización de superficies cuádricas y curvas}

\section{Elipsoide:}

Ecuación canónica:

$$
\frac{(x-i)^{2}}{a^{2}}+\frac{(y-j)^{2}}{b^{2}}+\frac{(z-k)^{2}}{c^{2}}=1
$$

Parametrización:

$$
s(u, v):\left\{\begin{array}{l}
x=i-a \cos u \operatorname{sen} v \\
y=j-b \operatorname{sen} u \operatorname{sen} v ; \quad u \in[0,2 \pi], v \in[0, \pi] \\
z=k-c \cos v
\end{array}\right.
$$

\section{Hiperboloide de una hoja:}

Ecuación canónica:

$$
\frac{(x-i)^{2}}{a^{2}}+\frac{(y-j)^{2}}{b^{2}}-\frac{(z-k)^{2}}{c^{2}}=1
$$

Parametrización:

$$
s(u, v):\left\{\begin{array}{l}
x=i-a \cos u \cosh v \\
y=j-b \operatorname{sen} u \cosh v ; \quad u \in[0,2 \pi], v \in \mathbb{R} \\
z=k-c \operatorname{senh} v
\end{array}\right.
$$




\section{Hiperboloide de dos hojas:}

Ecuación canónica:

$$
-\frac{(x-i)^{2}}{a^{2}}-\frac{(y-j)^{2}}{b^{2}}+\frac{(z-k)^{2}}{c^{2}}=1
$$

Parametrización hoja eje positivo:

$$
s(u, v):\left\{\begin{array}{l}
x=i+a \operatorname{senh} u \cos v \\
y=j+b \operatorname{senh} u \operatorname{sen} v ; \quad u \in[0,+\infty[, v \in[0,2 \pi] \\
z=k+c \cosh u
\end{array}\right.
$$

Parametrización hoja eje negativo:

$$
s(u, v):\left\{\begin{array}{l}
x=i-a \operatorname{senh} u \cos v \\
y=j-b \operatorname{senh} u \operatorname{sen} v ; \quad u \in[0,+\infty[, v \in[0,2 \pi] \\
z=k-c \cosh u
\end{array}\right.
$$

\section{Cono elíptico:}

Ecuación canónica:

$$
\frac{(x-i)^{2}}{a^{2}}+\frac{(y-j)^{2}}{b^{2}}-\frac{(z-k)^{2}}{c^{2}}=0
$$

Parametrización:

$$
s(u, v):\left\{\begin{array}{l}
x=i+\frac{a}{c} \operatorname{senh} u \cos v \\
y=j+\frac{b}{c} \operatorname{senh} u \operatorname{sen} v ; \quad u \in \mathbb{R}, v \in[0,2 \pi] \\
z=k+\operatorname{senh} u
\end{array}\right.
$$

\section{Paraboloide elíptico:}

Ecuación canónica:

$$
z-k=\frac{(x-i)^{2}}{a^{2}}+\frac{(y-j)^{2}}{b^{2}}
$$

Parametrización:

$$
s(u, v):\left\{\begin{array}{l}
x=u \\
y=v \\
z=\frac{(u-i)^{2}}{a^{2}}+\frac{(v-j)^{2}}{b^{2}}+k
\end{array} ; \quad u \in \mathbb{R}, v \in \mathbb{R}\right.
$$




\section{Paraboloide hiperbólico:}

Ecuación canónica:

$$
z-k=-\frac{(x-i)^{2}}{a^{2}}+\frac{(y-j)^{2}}{b^{2}}
$$

Parametrización:

$$
s(u, v):\left\{\begin{array}{l}
x=u \\
y=v \\
z=-\frac{(u-i)^{2}}{a^{2}}+\frac{(v-j)^{2}}{b^{2}}+k
\end{array} ; \quad u \in \mathbb{R}, v \in \mathbb{R}\right.
$$

\section{Curvas en 3D}

Elipse en al plano $x=k$

$$
\frac{(y-j)^{2}}{b^{2}}+\frac{(z-k)^{2}}{c^{2}}=1
$$

Usando $\operatorname{sen}^{2} x+\cos ^{2} x=1$, su parametrización estaría dada por:

$$
c(t):\left\{\begin{array}{l}
x=k \\
y=j+b \cos t \quad ; \quad t \in[0,2 \pi] \\
z=k+c \operatorname{sen} t
\end{array}\right.
$$

Hipérbola en al plano $x=k$

$$
\frac{(y-j)^{2}}{b^{2}}-\frac{(z-k)^{2}}{c^{2}}=1
$$

Usando $\sec ^{2} x-\tan ^{2} x=1$, su parametrización estaría dada por:

$$
c(t):\left\{\begin{array}{l}
x=k \\
y=j+b \sec t \quad ; \quad t \in\left[\frac{-\pi}{2}, \frac{3 \pi}{2}\right] \\
z=k+c \tan t
\end{array}\right.
$$

Parábola en al plano $x=k$

$$
y-j=\frac{(z-k)^{2}}{c^{2}}
$$


Su parametrización estaría dada por:

$$
c(t):\left\{\begin{array}{l}
x=k \\
y=\frac{(t-k)^{2}}{c^{2}}+j ; \quad t \in \mathbb{R} \\
z=t
\end{array}\right.
$$

\subsection{Conclusión}

La graficación en tres dimensiones es, sin duda, una de las grandes dificultades que afrontan los estudiantes de cursos de cálculo en varias variables, por lo que contar con un programa computacional gratuito, bastante amigable y didáctico es una valiosa ayuda, tanto para el profesor como para el alumno.

\section{Bibliografía}

[1] Larson, R. Edwards, B Cálculo 2 de varias variables. Mc Graw Hill. 2010.

[2] Mora, W Cálculo en varias variables. Revista Digital: Matemática, Educación e Internet. 2015.

[3] Geogebra Manual de GeoGebra 5.0. https://wiki.geogebra.org/es/Manual.

[4] Geogebra Tutoriales. https://wiki.geogebra.org/es/Tutoriales. 